\title{
Wave propagation in a infectious disease model with non-local diffusion
}

\author{
Yueling Cheng ${ }^{1 *}$ and Dianchen Lu'
}

\section{"Correspondence:}

1000003284@ujs.edu.cn

${ }^{1}$ Faculty of Science, Jiangsu

University, Zhenjiang, People's

Republic of China

\section{Springer}

\begin{abstract}
In this paper, we propose a nonlocal diffusion infectious disease model with nonlinear incidences and distributed delay to model the transmission of the epidemic. By a fixed point theorem and a limiting argument, we establish the existence of traveling wave solutions for the model. Meanwhile, we obtain the non-existence of traveling wave solutions for the model via two-sided Laplace transform. It is found that the threshold dynamics of traveling wave solutions are entirely determined by the basic reproduction number of the corresponding spatially-homogenous delayed differential system and the minimum wave speed. A typical example is given for supporting our abstract results. Moreover, the effect of the diffusive rate of the infected individuals on the minimum wave speed is discussed.
\end{abstract}

Keywords: Disease model; Nonlocal diffusion; Fixed point theorem; Two-sided Laplace transform

\section{Introduction}

With the rapid development of modern mathematical epidemiology, many mathematical models have been proposed to describe the transmission of communicable diseases. Among these models, one of the cornerstones is the classical Kermack-McKendrick SIR epidemic model [21-23]

$$
\left\{\begin{array}{l}
\dot{s}(t)=-\beta s(t) i(t), \\
\dot{i}(t)=\beta s(t) i(t)-\gamma i(t), \\
\dot{r}(t)=\gamma i(t),
\end{array}\right.
$$

where $s(t), i(t)$ and $r(t)$ denote the sizes of the susceptible, infected and removed individuals, respectively. The constant $\beta>0$ is the transmission coefficient and $\gamma>0$ represents the recovery rate. Kermack and McKendrick [21-23] proved that if the basic reproduction number $R_{0}=\beta / \gamma>1, i(t)$ first increases to its maximum and then decreases to zero and hence an epidemic occurs; if $R_{0}<1$, then $i(t)$ decreases to zero and an epidemic does not take place.

Due to large mobility of people within a country or even worldwide, the spatially uniform models are not sufficient to give a realistic picture of disease diffusion. Based on this

(c) The Author(s) 2019. This article is distributed under the terms of the Creative Commons Attribution 4.0 International License (http://creativecommons.org/licenses/by/4.0/), which permits unrestricted use, distribution, and reproduction in any medium, provided you give appropriate credit to the original author(s) and the source, provide a link to the Creative Commons license, and indicate if changes were made. 
concern, Hosono and Ilyas [18] introduced a reaction-diffusion epidemic model

$$
\left\{\begin{array}{l}
s_{t}(x, t)=d_{1} s_{x x}(x, t)-\beta s(x, t) i(x, t) \\
i_{t}(x, t)=d_{2} i_{x x}(x, t)+\beta s(x, t) i(x, t)-\gamma i(x, t)
\end{array}\right.
$$

where $d_{1}>0$ and $d_{2}>0$ are the diffusion rates for the susceptible and infected individuals, respectively. They showed that if the basic reproduction number $R_{0}=\beta / \gamma>1$ and the wave speed $c \geq c^{*}$ ( $c^{*}$ is the critical speed), system (1.2) admits a nontrivial and nonnegative traveling wave solution $(s(x+c t), i(x+c t))$ satisfying $s(\infty)=s_{\infty}<s_{-\infty}=s(-\infty)$ and $i( \pm \infty)=0$, while there exist no nontrivial and nonnegative traveling wave solutions if $R_{0} \leq 1$ or $0<c<c^{*}$.

Note that the bilinear incidence $\beta s i$ will be reasonable for a small number of infected, and it appears quite unrealistic that for a large $i$ this can still hold, see [7] for more details. Recently, nonlinear incidences have been introduced in epidemic models, see [3, $6,7,24-26,31,32]$. For example, Capasso and Serio [7] introduced a saturated incidence $\beta s i /(1+\alpha i)(\alpha>0)$ into epidemic models to prevent the unboundedness of the contact rate. Liu et al. [31,32] considered the effect of nonlinear incidence of the form $i^{p} s^{q}(p, q>0)$ on the behavior of epidemiological models. It has been suggested that time delay should be incorporated into epidemic models in applications [3, 6, 27, 29, 44, 46]. In fact, for many infectious diseases, an infected individual is unable to pass on the disease and a certain amount of biological development is necessary before one can infect others. This period is called the latent period. After the latent period, the individual passes into the infectious period which ends when one's symptoms appear and one is removed to the recovered class. The latent period is assumed to be a constant $\tau$. A susceptible becomes infective if one contacts the person who is infected $\tau$ time units ago. In particular, for some vectorborne diseases (such as those distributed by mosquitoes), Beretta and Takeuchi [6] introduced a distributed delay of the form $s(t) \int_{0}^{h} f(\tau) i(t-\tau) d \tau$ into the SIR model, where $h$ is the maximum time taken to become infectious and $f(\tau)$ denotes the fraction of a vector population in which the time taken to be infectious is $\tau$. Furthermore, $f(\tau)$ satisfies

$$
f(\tau)>0, f(\tau) \in C[0, h], \quad \int_{0}^{h} f(\tau) d \tau=1 .
$$

Bai and Zhang [3] investigated a reaction-diffusion model with nonlinear incidences and distributed delay

$$
\left\{\begin{array}{l}
s_{t}(x, t)=d_{1} s_{x x}(x, t)-\beta s(x, t) \int_{0}^{h} f(\tau) g(i(x, t-\tau)) d \tau, \\
i_{t}(x, t)=d_{2} i_{x x}(x, t)+\beta s(x, t) \int_{0}^{h} f(\tau) g(i(x, t-\tau)) d \tau-\gamma i(x, t),
\end{array}\right.
$$

where the nonlinear function $g$ satisfies two hypotheses: $(\mathrm{H} 1) g(i)$ is continuously differentiable and monotone increasing on $[0, \infty)$ with $g(0)=0 ;(\mathrm{H} 2) i / g(i)$ is monotone increasing on $(0, \infty)$ with $\lim _{i \rightarrow 0^{+}} i / g(i)=1$. The authors showed that (1.4) admits a nontrivial and nonnegative traveling wave solution $(s(x+c t), i(x+c t))$ satisfying $s(\infty)=s_{\infty}<s_{0}=s(-\infty)$, $i( \pm \infty)=0$ if $R_{0}>1$ and $c \geq c^{*}$. Moreover, they obtained that, if $0<c<c^{*}$ or $R_{0} \leq 1$, there exist no such traveling wave solutions. Recently, utilizing Schauder's fixed point theorem and the two-sided Laplace transform, Wang et al. [42], Xu [46], Zhang and Wang [54], 
Zhen et al. [55] investigated the existence and nonexistence of traveling wave solutions for a class of reaction-diffusion disease systems with nonlinear incidences and established some important existence and nonexistence theorems.

It is generally known that reaction-diffusion equations have been intensively studied to describe many phenomena in epidemiology and spatial ecology $[3,15,16,18,29,36$, $41,42,44-46,54,55]$. However, a nonlocal diffusion is better described as a long-range process rather than as a local one in many situations such as in population ecology, since the movements of individuals which cannot be limited to a small area are often free and random. During the past ten years, a special integral operator of the form

$$
J * u(x, t)-u(x, t)=\int_{-\infty}^{\infty} J(x-y)[u(y, t)-u(x, t)] d y
$$

has been widely used to model diffusion phenomena. Simultaneously, nonlocal diffusion problems have attracted much attention, see $[4,5,8-14,19,20,28,30,35,36,38,40$, $47-53,56,57]$ and the references therein.

In the present paper we propose a nonlocal diffusion infectious disease model with nonlinear incidences and distributed delay

$$
\left\{\begin{aligned}
s_{t}(x, t)= & d_{1} \int_{-\infty}^{\infty} J(x-y)[s(y, t)-s(x, t)] d y \\
& -\beta s(x, t) \int_{0}^{h} f(\tau) g(i(x, t-\tau)) d \tau, \\
i_{t}(x, t)= & d_{2} \int_{-\infty}^{\infty} J(x-y)[i(y, t)-i(x, t)] d y \\
& +\beta s(x, t) \int_{0}^{h} f(\tau) g(i(x, t-\tau)) d \tau-\gamma i(x, t)
\end{aligned}\right.
$$

to model the propagation of the epidemic. In (1.5), the integral operators $J * s(x, t)-s(x, t)$ and $J * i(x, t)-i(x, t)$ mean that the rate of susceptible and infected individuals in position $x$ and at time $t$ depend on the influence of neighboring $s(x, t)$ and $i(x, t)$ in all other positions $y$ [56,57]. Model (1.5) with nonlinear incidences and distributed delay describes the spread of an infectious disease (involving only susceptible and infected individuals) transmitted by a vector (e.g., mosquitoes) after a latent period [39]. Throughout this paper, we always assume that $f(\tau)$ satisfies (1.3), the nonlinear function $g$ and the kernel function $J$ satisfy

(A1) $g(0)=0, g^{\prime}(i)>0$ and $g^{\prime \prime}(i) \leq 0$ for $i \geq 0$;

(A2) $g(i) / i$ is continuously differentiable, nonincreasing for $i>0$ and $\lim _{i \rightarrow \infty} g(i) / i=0$;

(A3) $J \in C^{1}(\mathbb{R}), J(y)=J(-y) \geq 0, \int_{\mathbb{R}} J(y) d y=1, J$ is compactly supported;

(A4) $\lim _{\lambda \rightarrow \infty} \lambda^{-1} \int_{\mathbb{R}} J(y) e^{-\lambda y} d y=\infty$ and $\int_{\mathbb{R}} J(x) e^{\lambda x} d x<+\infty$ for all $\lambda>0$.

Assumptions (A1)-(A4) have been used in the literature, one can refer to [3, 17, 26, 49, $50,56,57]$. The aim of the current paper is to study the existence and nonexistence of the nontrivial and nonnegative traveling wave solutions of the form

$$
(s(x+c t), i(x+c t))=(s(z), i(z)),
$$

where $z=x+c t$ is the moving coordinate and $c>0$ is the wave speed. Substituting (1.6) into (1.5) yields

$$
\left\{\begin{aligned}
c s^{\prime}(z)= & d_{1} \int_{-\infty}^{\infty} J(y)[s(z-y)-s(z)] d y-\beta s(z) \int_{0}^{h} f(\tau) g(i(z-c \tau)) d \tau \\
c i^{\prime}(z)= & d_{2} \int_{-\infty}^{\infty} J(y)[i(z-y)-i(z)] d y+\beta s(z) \int_{0}^{h} f(\tau) g(i(z-c \tau)) d \tau \\
& -\gamma i(z) .
\end{aligned}\right.
$$


We are going to find the solutions of (1.7) which satisfy the asymptotic boundary conditions

$$
\begin{aligned}
& \lim _{z \rightarrow \infty} s(z):=s(\infty)=s_{\infty}<\lim _{z \rightarrow-\infty} s(z):=s(-\infty)=s_{0}, \\
& \lim _{z \rightarrow \pm \infty} i(z):=i( \pm \infty)=0
\end{aligned}
$$

where $s_{0}>0$ refers to the size of the susceptible individuals at the beginning of the epidemic and $s_{\infty} \geq 0$ stands for the size of the susceptible individuals after the epidemic.

We should point out that our model is different from model (1.4), which can be viewed as a first order approximation of model (1.5) by Fourier transform and Taylor formula $[19,20]$. In [3], under the traveling wave transformation, (1.4) was reduced to a system of second order ordinary differential equations (ODEs). By the general theory of ODEs, the solution of this system can be written as integral forms on the real line. By introducing an auxiliary system and applying the Schauder's fixed point theorem together with a limiting argument, Bai and Zhang [3] obtained the existence of a traveling wave solution for their model directly. Notice that (1.5) has no monotone semi-flow due to the introduction of nonlocal diffusion, the method used in [3] cannot be effective for (1.5). Moreover, other traditional methods such as the method of monotone iteration together with upper-lower solutions [45] and the shooting method [18] cannot be applied any more. To obtain the existence theorem for (1.5), we construct an invariant cone of initial functions defined on a large spatial domain and apply Schauder's fixed point theorem on this cone and a limiting argument (motivated by [30, 42, 49, 50,54]) to establish the existence of a solution for the wave system. Note also that in [3], the authors investigated the nonexistence of the solutions for their model for the cases $R_{0}<1$ and $R_{0}=1$, simultaneously. However, in the proof of Theorem 1.3 in [3], the last inequality does not hold when $R_{0}=1$. Here in the proof of the nonexistence theorem for $R_{0} \leq 1$, we divide it into two subcases, namely, $R_{0}<1$ and $R_{0}=1$. We hope that the techniques adopted here can be used to study other delayed general nonlocal diffusion systems.

The rest of the paper is organized as follows. In Sect. 2, the existence of traveling wave solutions of (1.5) is established. In Sect. 3, the nonexistence of traveling wave solutions of (1.5) is obtained. In Sect. 4, we give an example to illustrate the theoretical results and make a brief conclusion.

\section{Existence of traveling waves}

\subsection{Preliminaries}

Linearizing the second equation in $(1.7)$ at $\left(s_{0}, 0\right)$ and using $g(0)=0$, we have

$$
d_{2} \int_{-\infty}^{\infty} J(y)[i(z-y)-i(z)] d y-c i^{\prime}(z)+\beta s_{0} g^{\prime}(0) \int_{0}^{h} f(\tau) i(z-c \tau) d \tau-\gamma i(z)=0 .
$$

Substituting $i(z)=e^{\lambda z}$ into $(2.1)$ yields

$$
\Theta(\lambda, c):=d_{2} \int_{-\infty}^{\infty} J(y)\left(e^{-\lambda y}-1\right) d y-c \lambda+\beta s_{0} g^{\prime}(0) \int_{0}^{h} f(\tau) e^{-\lambda c \tau} d \tau-\gamma=0 .
$$

Lemma 2.1 Assume that $R_{0}:=\beta s_{0} g^{\prime}(0) / \gamma>1$. Then there exist $c^{*}>0$ and $\lambda^{*}>0$ such that

$$
\Theta\left(\lambda^{*}, c^{*}\right)=0 \text { and } \Theta_{\lambda}\left(\lambda^{*}, c^{*}\right)=0 .
$$


Furthermore, the following alternatives hold:

(i) If $0<c<c^{*}$, then $\Theta(\lambda, c)>0$ for $\lambda \in[0, \infty)$;

(ii) If $c>c^{*}$, then equation $\Theta(\lambda, c)=0$ has two positive real roots $\lambda_{1}(c):=\lambda_{1}$ and $\lambda_{2}(c):=\lambda_{2}$ with $0<\lambda_{1}<\lambda^{*}<\lambda_{2}<\infty$ such that

$$
\begin{cases}\Theta(\lambda, c)>0, & \lambda \in\left[0, \lambda_{1}\right) \cup\left(\lambda_{2}, \infty\right), \\ \Theta(\lambda, c)<0, & \lambda \in\left(\lambda_{1}, \lambda_{2}\right) .\end{cases}
$$

Proof Obviously, $\Theta(0, c)=\beta s_{0} g^{\prime}(0)-\gamma>0$. Using (A4), we have $\Theta(\infty, c)=\infty$ for each fixed $c>0$. Meanwhile, by (A3), we derive for $\lambda>0$ and $c>0$ that

$$
\begin{aligned}
\Theta_{c}(\lambda, c) & =-\lambda-\lambda \beta s_{0} g^{\prime}(0) \int_{0}^{h} \tau f(\tau) e^{-\lambda c \tau} d \tau<0, \\
\Theta(\lambda, 0) & =d_{2} \int_{-\infty}^{\infty} J(y)\left(e^{-\lambda y}-1\right) d y+\beta s_{0} g^{\prime}(0)-\gamma \\
& \geq-d_{2} \lambda \int_{-\infty}^{\infty} y J(y) d y+\beta s_{0} g^{\prime}(0)-\gamma \\
& =\beta s_{0} g^{\prime}(0)-\gamma>0 \\
\Theta(\lambda, \infty) & =-\infty \\
\Theta_{\lambda}(0, c) & =-c-c \beta s_{0} g^{\prime}(0) \int_{0}^{h} \tau f(\tau) d \tau<0, \\
\Theta_{\lambda \lambda}(\lambda, c) & =d_{2} \int_{-\infty}^{\infty} y^{2} J(y) e^{-\lambda y} d y+\beta s_{0} g^{\prime}(0) c^{2} \int_{0}^{h} \tau^{2} f(\tau) e^{-\lambda c \tau} d \tau>0 .
\end{aligned}
$$

By the intermediate value theorem, monotonicity and convexity properties, there exists a positive point $\left(\lambda^{*}, c^{*}\right)$ such that

$$
\Theta\left(\lambda^{*}, c^{*}\right)=0 \text { and } \Theta_{\lambda}\left(\lambda^{*}, c^{*}\right)=0
$$

Since $\Theta_{c}(\lambda, c)<0$, we get that $\Theta\left(\lambda^{*}, c\right)>\Theta\left(\lambda^{*}, c^{*}\right)=0$ for any $0<c<c^{*}$. Using the convexity of $\Theta(\lambda, c)$ with respect to $\lambda$, we have

$$
\Theta\left(\lambda_{1}, c\right) \geq \Theta\left(\lambda^{*}, c\right)=0 \quad \text { for } \lambda \in[0, \infty)
$$

Due to $\Theta_{c}(\lambda, c)<0$, we have that $\Theta\left(\lambda^{*}, c\right)<\Theta\left(\lambda^{*}, c^{*}\right)=0$ for any $c>c^{*}$. Then by $\Theta(0, c)>0$, $\Theta_{\lambda \lambda}(\lambda, c)>0$ and $\Theta(\lambda, c) \rightarrow \infty$ as $\lambda \rightarrow \infty$, we obtain that the equation $\Theta(\lambda, c)=0$ has two positive roots $\lambda_{1}(c):=\lambda_{1}$ and $\lambda_{2}(c):=\lambda_{2}$ with $0<\lambda_{1}<\lambda^{*}<\lambda_{2}<\infty$ such that

$$
\Theta(\lambda, c) \begin{cases}>0, & \lambda \in\left[0, \lambda_{1}\right) \cup\left(\lambda_{2}, \infty\right), \\ <0, & \lambda \in\left(\lambda_{1}, \lambda_{2}\right) .\end{cases}
$$

The proof of this lemma is finished.

In the remainder of this section, we always assume that $R_{0}>1$ and $c>c^{*}$. 


\subsection{Key lemmas}

Set

$$
q(i):=\beta s_{0} \frac{g(i)}{i}, \quad i>0,
$$

then $q(i)$ is a continuous differential function for $i>0$ (see (A2)). A direct computation yields

$$
\lim _{i \rightarrow 0^{+}} q(i)=\lim _{i \rightarrow 0^{+}} \beta s_{0} \frac{g(i)}{i}=\beta s_{0} g^{\prime}(0)>\gamma
$$

and

$$
\lim _{i \rightarrow \infty} q(i)=\lim _{i \rightarrow \infty} \beta s_{0} \frac{g(i)}{i}=0<\gamma,
$$

where we have used $R_{0}>1$ and (A2). So there exists a positive constant $i_{0}$ such that

$$
\beta s_{0} g\left(i_{0}\right)=\gamma i_{0}
$$

For $z \in \mathbb{R}$, define the following nonnegative continuous functions:

$$
\begin{aligned}
& \bar{s}(z):=s_{0}, \\
& \bar{i}(z):= \begin{cases}e^{\lambda_{1} z}, & z<\lambda_{1}^{-1} \ln i_{0}, \\
i_{0}, & z \geq \lambda_{1}^{-1} \ln i_{0},\end{cases} \\
& \underline{s}(z):= \begin{cases}s_{0}-\mu^{-1} e^{\mu z}, \quad z<\mu^{-1} \ln \left(\mu s_{0}\right), \\
0, & z \geq \mu^{-1} \ln \left(\mu s_{0}\right),\end{cases} \\
& \underline{i}(z):= \begin{cases}e^{\lambda_{1} z}\left(1-M e^{\alpha z}\right), & z<-\alpha^{-1} \ln M, \\
0, & z \geq-\alpha^{-1} \ln M,\end{cases}
\end{aligned}
$$

where $\lambda_{1}$ is the smaller positive real root of (2.2) and $\mu, M, \alpha$ are all positive constants which will be determined later.

Lemma 2.2 The function $\bar{s}(z)$ satisfies

$$
d_{1} \int_{-\infty}^{\infty} J(y) \bar{s}(z-y) d y-d_{1} \bar{s}(z)-c \bar{s}^{\prime}(z)-\beta \bar{s}(z) \int_{0}^{h} f(\tau) g(\underline{i}(z-c \tau)) d \tau \leq 0
$$

for all $z \in \mathbb{R}$.

Proof The proof is trivial, so it is omitted.

Lemma 2.3 The function $\bar{i}(z)$ satisfies

$$
d_{2} \int_{-\infty}^{\infty} J(y) \bar{i}(z-y) d y-d_{2} \bar{i}(z)-c \bar{i}^{\prime}(z)+\beta \bar{s}(z) \int_{0}^{h} f(\tau) g(\bar{i}(z-c \tau)) d \tau-\gamma \bar{i}(z) \leq 0
$$

for all $z \neq z_{1}:=\lambda_{1}^{-1} \ln i_{0}$. 
Proof It follows from (A1)-(A3) that

$$
\int_{-\infty}^{\infty} J(y) \bar{i}(z-y) d y \leq \min \left\{e^{\lambda_{1} z} \int_{-\infty}^{\infty} J(y) e^{-\lambda_{1} y} d y, i_{0}\right\}, \quad z \in \mathbb{R}
$$

and

$$
g(\bar{i}(z-c \tau))=g(\bar{i}(z-c \tau))-g(0) \leq g^{\prime}(0) \bar{i}(z-c \tau) .
$$

If $z<z_{1}$, then $\bar{i}(z)=e^{\lambda_{1} z}$. From (2.4), (2.5) and Lemma 2.1, we obtain that

$$
\begin{aligned}
& d_{2} \int_{-\infty}^{\infty} J(y) \bar{i}(z-y) d y-d_{2} \bar{i}(z)-c \bar{i}^{\prime}(z)+\beta \bar{s}(z) \int_{0}^{h} f(\tau) g(\bar{i}(z-c \tau)) d \tau-\gamma \bar{i}(z) \\
& \quad \leq d_{2} \int_{-\infty}^{\infty} J(y) \bar{i}(z-y) d y-d_{2} \bar{i}(z)-c \bar{i}^{\prime}(z)+\beta s_{0} g^{\prime}(0) \int_{0}^{h} f(\tau) \bar{i}(z-c \tau) d \tau-\gamma \bar{i}(z) \\
& \quad=e^{\lambda_{1} z}\left[d_{2} \int_{-\infty}^{\infty} J(y)\left(e^{-\lambda_{1} y}-1\right) d y-c \lambda_{1}+\beta s_{0} g^{\prime}(0) \int_{0}^{h} f(\tau) e^{-\lambda_{1} c \tau} d \tau-\gamma\right] \\
& \quad=e^{\lambda_{1} z} \Theta\left(\lambda_{1}, c\right) \\
& \quad=0 .
\end{aligned}
$$

If $z>z_{1}$, then $\bar{i}(z)=i_{0}$. By (2.3), (A1) and the definition of $\bar{i}(z)$, we have that

$$
\begin{aligned}
& d_{2} \int_{-\infty}^{\infty} J(y) \bar{i}(z-y) d y-d_{2} \bar{i}(z)-c \bar{i}^{\prime}(z)+\beta \bar{s}(z) \int_{0}^{h} f(\tau) g(\bar{i}(z-c \tau)) d \tau-\gamma \bar{i}(z) \\
& \quad \leq \beta s_{0} g\left(i_{0}\right)-\gamma i_{0} \\
& \quad=0 .
\end{aligned}
$$

The proof of this lemma is completed.

Lemma 2.4 Let $\mu \in\left(0, \lambda_{1}\right)$ be sufficiently small. Then the function $\underline{s}(z)$ satisfies

$$
d_{1} \int_{-\infty}^{\infty} J(y) \underline{s}(z-y) d y-d_{1} \underline{s}(z)-c \underline{s}^{\prime}(z)-\beta \underline{s}(z) \int_{0}^{h} f(\tau) g(\bar{i}(z-c \tau)) d \tau \geq 0
$$

for all $z \neq z_{2}:=\mu^{-1} \ln \left(\mu s_{0}\right)$.

Proof If $z>z_{2}$, then $\underline{s}(z)=0$, which implies that (2.6) holds.

Note that $z_{2}=\mu^{-1} \ln \left(\mu s_{0}\right)<\lambda_{1}^{-1} \ln i_{0}=z_{1}$ as $\mu \rightarrow 0^{+}$. If $z<z_{2}<z_{1}$, then

$$
\underline{s}(z)=s_{0}-\mu^{-1} e^{\mu z} \text { and } \bar{i}(z)=e^{\lambda_{1} z} .
$$


It follows from (2.5) and (2.7) that

$$
\begin{aligned}
d_{1} \int_{-\infty}^{\infty} J(y) \underline{s}(z-y) d y-d_{1} \underline{s}(z)-c \underline{s}^{\prime}(z)-\beta \underline{s}(z) \int_{0}^{h} f(\tau) g(\bar{i}(z-c \tau)) d \tau \\
\geq d_{1} \int_{-\infty}^{\infty} J(y) \underline{s}(z-y) d y-d_{1} \underline{s}(z)-c \underline{s}^{\prime}(z)-\beta \underline{s}(z) g^{\prime}(0) \int_{0}^{h} f(\tau) \bar{i}(z-c \tau) d \tau \\
=-d_{1} \mu^{-1} e^{\mu z} \int_{-\infty}^{\infty} J(y) e^{-\mu y} d y+d_{1} \mu^{-1} e^{\mu z}+c e^{\mu z} \\
\quad-\beta\left(s_{0}-\mu^{-1} e^{\mu z}\right) g^{\prime}(0) \int_{0}^{h} f(\tau) e^{\lambda_{1}(z-c \tau)} d \tau \\
\geq e^{\mu z}\left[-d_{1} \mu^{-1} \int_{-\infty}^{\infty} J(y)\left(e^{-\mu y}-1\right) d y+c-\beta s_{0} g^{\prime}(0) e^{\left(\lambda_{1}-\mu\right) z}\right] \\
\geq e^{\mu z}\left[-d_{1} \mu^{-1} \int_{-\infty}^{\infty} J(y)\left(e^{-\mu y}-1\right) d y+c-\beta s_{0} g^{\prime}(0)\left(\mu s_{0}\right)^{\frac{\lambda_{1}-\mu}{\mu}}\right]
\end{aligned}
$$

where we have used the fact that $e^{\left(\lambda_{1}-\mu\right) z}<\left(\mu s_{0}\right)^{\frac{\lambda_{1}-\mu}{\mu}}$ for $z<z_{2}$ and the assumption that $0<\mu<\lambda_{1}$. Since $\lim _{\mu \rightarrow 0^{+}}\left(\mu s_{0}\right)^{\frac{\lambda_{1}-\mu}{\mu}}=0$ and $\lim _{\mu \rightarrow 0^{+}} \mu^{-1} \int_{\mathbb{R}} J(y)\left(e^{-\mu y}-1\right) d y=0$, we have for sufficiently small $\mu>0$ that

$$
-d_{1} \mu^{-1} \int_{-\infty}^{\infty} J(y)\left(e^{-\mu y}-1\right) d y+c-\beta s_{0} g^{\prime}(0)\left(\mu s_{0}\right)^{\frac{\lambda_{1}-\mu}{\mu}}>0 .
$$

This ends this lemma.

Lemma 2.5 Suppose that $0<\alpha<\min \left\{\mu, \lambda_{1}, \lambda_{2}-\lambda_{1}\right\}$ and $M>1$ is sufficiently large. Then the function $\underline{i}(z)$ satisfies

$$
\begin{aligned}
& d_{2} \int_{-\infty}^{\infty} J(y) \underline{i}(z-y) d y-d_{2} \underline{i}(z)-\underline{c}^{\prime}(z) \\
& \quad+\beta \underline{s}(z) \int_{0}^{h} f(\tau) g(\underline{i}(z-c \tau)) d \tau-\gamma \underline{i}(z) \geq 0
\end{aligned}
$$

for all $z \neq z_{3}:=-\alpha^{-1} \ln M$

Proof If $z>z_{3}$, then $\underline{i}(z)=0, \underline{s}(z) \geq 0, \int_{\mathbb{R}} J(y) \underline{i}(z-y) d y \geq 0$ and $g(\underline{i}(z-c \tau)) \geq 0$. Obviously, (2.10) holds.

If $z<z_{3}$, then $\underline{i}(z)=e^{\lambda_{1} z}\left(1-M e^{\alpha z}\right)$. Let $M_{1}>1$ be large enough such that $-\alpha^{-1} \ln M_{1}+1=$ $z_{2}$. Choose $M \geq M_{1}$, then $\underline{s}(z)=s_{0}-\mu^{-1} e^{\mu z}$ for $z<z_{3}<z_{2}$. Thus (2.10) is equivalent to

$$
\begin{aligned}
& \beta s_{0} g^{\prime}(0) \int_{0}^{h} f(\tau) \underline{i}(z-c \tau) d \tau-\beta \underline{s}(z) \int_{0}^{h} f(\tau) g(\underline{i}(z-c \tau)) d \tau \\
& \quad \leq d_{2} \int_{-\infty}^{\infty} J(y) \underline{i}(z-y) d y-d_{2} \underline{i}(z)-c \underline{i}^{\prime}(z)+\beta s_{0} g^{\prime}(0) \int_{0}^{h} f(\tau) \underline{i}(z-c \tau) d \tau-\gamma \underline{i}(z),
\end{aligned}
$$


which gives

$$
\begin{gathered}
\beta s_{0} \int_{0}^{h} f(\tau)\left[g^{\prime}(0) \underline{i}(z-c \tau)-g(\underline{i}(z-c \tau))\right] d \tau \\
+\beta \mu^{-1} e^{\mu z} \int_{0}^{h} f(\tau) g(\underline{i}(z-c \tau)) d \tau \\
\leq \Theta\left(\lambda_{1}, c\right) e^{\lambda_{1} z}-M \Theta\left(\lambda_{1}+\alpha, c\right) e^{\left(\lambda_{1}+\alpha\right) z} .
\end{gathered}
$$

By (A2), we know that, for any $\hat{\varepsilon} \in(0,1)$, there exists a small positive constant $\delta_{0}$ such that

$$
\frac{g(i)}{i} \geq g^{\prime}(0)-\hat{\varepsilon} \quad \text { for } 0<i<\delta_{0} .
$$

Thus for $0<i<\delta_{0}$, one has that

$$
g^{\prime}(0) i-g(i)=i\left[g^{\prime}(0)-\frac{g(i)}{i}\right] \leq i \hat{\varepsilon} \leq\left(\frac{i+\hat{\varepsilon}}{2}\right)^{2}
$$

Since (2.13) holds for any sufficiently small $\hat{\varepsilon} \in(0,1)$, we obtain that

$$
g^{\prime}(0) i-g(i) \leq i^{2} \quad \text { for } 0<i<\delta_{0} .
$$

In order to ensure (2.11) holds, it suffices to prove that

$$
\begin{aligned}
& \beta s_{0} \int_{0}^{h} f(\tau) \underline{i}^{2}(z-c \tau) d \tau+\beta \mu^{-1} g^{\prime}(0) e^{\mu z} \int_{0}^{h} f(\tau) \underline{i}(z-c \tau) d \tau \\
& \quad \leq-M \Theta\left(\lambda_{1}+\alpha, c\right) e^{\left(\lambda_{1}+\alpha\right) z} .
\end{aligned}
$$

Further enlarging the left-hand side of (2.14) by $\underline{i}(z)<e^{\lambda_{1} z}$ gives that

$$
\beta s_{0} e^{\left(\lambda_{1}-\alpha\right) z}+\beta \mu^{-1} g^{\prime}(0) e^{(\mu-\alpha) z} \leq-M \Theta\left(\lambda_{1}+\alpha, c\right) .
$$

Choose $0<\alpha<\lambda_{2}-\lambda_{1}$, then $\Theta\left(\lambda_{1}+\alpha, c\right)<0$ (see Lemma 2.1). We infer from (2.15) that

$$
M \geq \frac{\beta s_{0} e^{\left(\lambda_{1}-\alpha\right) z}+\beta \mu^{-1} g^{\prime}(0) e^{(\mu-\alpha) z}}{-\Theta\left(\lambda_{1}+\alpha, c\right)} .
$$

Since $z<z_{2}<0$ and $0<\alpha<\min \left\{\mu, \lambda_{1}\right\}$, one knows that

$$
e^{\left(\lambda_{1}-\alpha\right) z}<1, \quad e^{(\mu-\alpha) z}<1
$$

Then (2.15) holds if we choose that

$$
M \geq \max \left\{\frac{\beta s_{0}+\beta \mu^{-1} g^{\prime}(0)}{-\Theta\left(\lambda_{1}+\alpha, c\right)}+1, M_{1}\right\} .
$$

The claim of this lemma is shown. 


\subsection{Existence of solution for (1.7) on a closed bounded interval}

Now we construct a closed bounded set

$$
\Gamma_{l}=\left\{\begin{array}{l|l}
(\phi(\cdot), \varphi(\cdot)) \in C\left([-l, l], \mathbb{R}^{2}\right) & \begin{array}{l}
\phi(-l)=\underline{s}(-l), \\
\varphi(-l)=\underline{i}(-l), \\
\underline{s}(z) \leq \phi(z) \leq s_{0}, \\
\underline{i}(z) \leq \varphi(z) \leq \bar{i}(z) \\
\text { for any } z \in[-l, l]
\end{array}
\end{array}\right\},
$$

where $l$ is a constant satisfying $l>\rho:=\max \left\{\left|z_{3}\right|, r, c h\right\} \quad(r$ is the radius of $\operatorname{supp} J)$. For $(\phi(z), \varphi(z)) \in C\left([-l, l], \mathbb{R}^{2}\right)$, define

$$
\hat{\phi}(z)=\left\{\begin{array}{ll}
\phi(l), & z>l, \\
\phi(z), & |z| \leq l, \\
\underline{s}(z), & z<-l,
\end{array} \quad \hat{\varphi}(z)= \begin{cases}\varphi(l), & z>l, \\
\varphi(z), & |z| \leq l, \\
\underline{i}(z), & z<-l .\end{cases}\right.
$$

Consider the initial value problem

$$
\begin{aligned}
c s^{\prime}(z)= & d_{1} \int_{-\infty}^{\infty} J(y) \hat{\phi}(z-y) d y+\varrho \phi(z)-\left(d_{1}+\varrho\right) s(z) \\
& -\beta \phi(z) \int_{0}^{h} f(\tau) g(\hat{\varphi}(z-c \tau)) d \tau, \\
c i^{\prime}(z)= & d_{2} \int_{-\infty}^{\infty} J(y) \hat{\varphi}(z-y) d y+\beta \phi(z) \int_{0}^{h} f(\tau) g(\hat{\varphi}(z-c \tau)) d \tau \\
& -\left(d_{2}+\gamma\right) i(z)
\end{aligned}
$$

on $[-l, l]$ with

$$
s(-l)=\underline{s}(-l), \quad i(-l)=\underline{i}(-l),
$$

where $\varrho$ is a constant satisfying $\varrho>\beta g\left(i_{0}\right)$. It is easy to verify that

$$
\underline{s}(z) \leq \hat{\phi}(z) \leq s_{0}, \quad \underline{i}(z) \leq \hat{\varphi}(z) \leq \bar{i}(z) \quad \text { for } z \in \mathbb{R} .
$$

By the general theory of ODEs, (2.16)-(2.18) admits a unique solution $\left(s_{l}(z), i_{l}(z)\right) \in$ $C^{1}\left([-l, l], \mathbb{R}^{2}\right)$. We define an operator $\mathcal{F}=\left(\mathcal{F}_{1}, \mathcal{F}_{2}\right): \Gamma_{l} \mapsto C\left([-l, l], \mathbb{R}^{2}\right)$ as follows:

$$
\mathcal{F}_{1}(\phi, \varphi)(z):=s_{l}(z), \quad \mathcal{F}_{2}(\phi, \varphi)(z):=i_{l}(z), \quad z \in[-l, l] .
$$

Proposition 2.1 The operator $\mathcal{F}=\left(\mathcal{F}_{1}, \mathcal{F}_{2}\right)$ maps $\Gamma_{l}$ into $\Gamma_{l}$. 
Proof Due to $\varrho>\beta g\left(i_{0}\right)$ and $\hat{\varphi}(z) \leq i_{0}$, we obtain that $\varrho \phi(z)-\beta \phi(z) \int_{0}^{h} f(\tau) g(\hat{\varphi}(z-c \tau)) d \tau$ is increasing with respect to $\phi$. Then we derive from Lemmas 2.2, 2.4 and (2.19) that

$$
\begin{aligned}
& d_{1} \int_{-\infty}^{\infty} J(y) \hat{\phi}(z-y) d y-c \bar{s}^{\prime}(z)+\varrho \phi(z)-\left(d_{1}+\varrho\right) \bar{s}(z) \\
& \quad-\beta \phi(z) \int_{0}^{h} f(\tau) g(\hat{\varphi}(z-c \tau)) d \tau \\
& \leq d_{1} \int_{-\infty}^{\infty} J(y) \bar{s}(z)(z-y) d y-c \bar{s}^{\prime}(z)+\varrho \bar{s}(z)-\left(d_{1}+\varrho\right) \bar{s}(z) \\
& \quad-\beta \bar{s}(z) \int_{0}^{h} f(\tau) g(\underline{i}(z-c \tau)) d \tau \\
& \leq 0, \quad z \in[-l, l],
\end{aligned}
$$

and

$$
\begin{aligned}
& d_{1} \int_{-\infty}^{\infty} J(y) \hat{\phi}(z-y) d y-c \underline{s}^{\prime}(z)+\varrho \phi(z)-\left(d_{1}+\varrho\right) \underline{s}(z) \\
& \quad-\beta \phi(z) \int_{0}^{h} f(\tau) g(\hat{\varphi}(z-c \tau)) d \tau \\
& \geq d_{1} \int_{-\infty}^{\infty} J(y) \underline{s}(z)(z-y) d y-c \underline{s}^{\prime}(z)+\varrho \underline{s}(z)-\left(d_{1}+\varrho\right) \underline{s}(z) \\
& \quad-\beta \underline{s}(z) \int_{0}^{h} f(\tau) g(\bar{i}(z-c \tau)) d \tau \\
& \geq 0, \quad z \in\left[-l, z_{2}\right) \cup\left(z_{2}, l\right] .
\end{aligned}
$$

By Lemmas 2.3, 2.5 and (2.19), we have that

$$
\begin{aligned}
c \underline{i}^{\prime}(z) & -d_{2} \int_{-\infty}^{\infty} J(y) \hat{\varphi}(z-y) d y-\beta \phi(z) \int_{0}^{h} f(\tau) g(\hat{\varphi}(z-c \tau)) d \tau \\
& +\left(d_{2}+\gamma\right) \underline{i}(z) \\
\leq & c \underline{i}^{\prime}(z)-d_{2} \int_{-\infty}^{\infty} J(y) \underline{i}(z-y) d y-\beta \underline{s}(z) \int_{0}^{h} f(\tau) g(\underline{i}(z-c \tau)) d \tau \\
& +\left(d_{2}+\gamma\right) \underline{i}(z) \\
\leq & 0, \quad z \in\left[-l, z_{3}\right) \cup\left(z_{3}, l\right],
\end{aligned}
$$

and

$$
\begin{aligned}
& c_{\bar{i}^{\prime}}(z)-d_{2} \int_{-\infty}^{\infty} J(y) \hat{\varphi}(z-y) d y-\beta \phi(z) \int_{0}^{h} f(\tau) g(\hat{\varphi}(z-c \tau)) d \tau+\left(d_{2}+\gamma\right) \bar{i}(z) \\
& \quad \geq c \bar{i}^{\prime}(z)-d_{2} \int_{-\infty}^{\infty} J(y) \bar{i}(z-y) d y-\beta s_{0} \int_{0}^{h} f(\tau) g(\bar{i}(z-c \tau)) d \tau+\left(d_{2}+\gamma\right) \bar{i}(z) \\
& \quad \geq 0, \quad z \in\left[-l, z_{1}\right) \cup\left(z_{1}, l\right] .
\end{aligned}
$$


In view of

$$
\underline{s}(-l)=s_{l}(-l) \leq \bar{s}(-l), \quad \underline{i}(-l)=i_{l}(-l) \leq \bar{i}(-l),
$$

(2.20)-(2.23), comparison theorem and the continuity of $\bar{s}(z), \bar{i}(z), \underline{s}(z), \underline{i}(z), s_{l}(z), i_{l}(z)$, we get that

$$
\underline{s}(z) \leq s_{l}(z) \leq \bar{s}(z), \quad \underline{i}(z) \leq i_{l}(z) \leq \bar{i}(z) \quad \text { for } z \in[-l, l] .
$$

The proof of this proposition is finished.

Proposition 2.2 The operator $\mathcal{F}=\left(\mathcal{F}_{1}, \mathcal{F}_{2}\right): \Gamma_{l} \mapsto \Gamma_{l}$ is completely continuous with respect to the supremum norm in $C\left([-l, l], \mathbb{R}^{2}\right)$.

Proof We first show that $\mathcal{F}$ is compact. Let $\left(s_{l}(z), i_{l}(z)\right)$ be the solution to (2.16)-(2.18). Since it is of class $C^{1}\left([-l, l], \mathbb{R}^{2}\right), s_{l}^{\prime}(z)$ and $i_{l}^{\prime}(z)$ are bounded on $[-l, l]$. Hence, by the definition of $\Gamma_{l}$, we obtain that the operator $\mathcal{F}$ is compact.

We next prove that $\mathcal{F}$ is continuous. Assume that $\left(\phi_{1}(z), \varphi_{1}(z)\right) \in \Gamma_{l}$ and $\left(\phi_{2}(z), \varphi_{2}(z)\right) \in$ $\Gamma_{l}$ for $z \in[-l, l]$. Equation (2.16) can be written as

$$
s_{l}(z)=\underline{s}(-l) e^{-\frac{d_{1}+Q}{c}(l+z)}+\frac{1}{c} \int_{-l}^{z} e^{-\frac{d_{1}+Q}{c}(z-\eta)} H_{1}(\phi, \varphi)(\eta) d \eta,
$$

where

$$
H_{1}(\phi, \varphi)(\eta)=d_{1} \int_{-\infty}^{\infty} J(y) \hat{\phi}(\eta-y) d y+\varrho \phi(\eta)-\beta \phi(\eta) \int_{0}^{h} f(\tau) g(\hat{\varphi}(\eta-c \tau)) d \tau .
$$

A direct computation gives that

$$
\begin{aligned}
\left|H_{1}\left(\phi_{1}, \varphi_{1}\right)(\eta)-H_{1}\left(\phi_{2}, \varphi_{2}\right)(\eta)\right| & \mid d_{1} \int_{-\infty}^{\infty} J(\eta-y)\left[\hat{\phi}_{1}(y)-\hat{\phi}_{2}(y)\right] d y+\varrho\left[\phi_{1}(\eta)-\phi_{2}(\eta)\right] \\
& -\beta\left[\phi_{1}(\eta) \int_{0}^{h} f(\tau) g\left(\hat{\varphi}_{1}(\eta-c \tau)\right) d \tau-\phi_{2}(\eta) \int_{0}^{h} f(\tau) g\left(\hat{\varphi}_{2}(\eta-c \tau)\right) d \tau\right] \mid \\
\leq & d_{1}\left|\int_{-l}^{l} J(\eta-y)\left[\phi_{1}(y)-\phi_{2}(y)\right] d y\right|+d_{1}\left|\int_{l}^{\infty} J(\eta-y)\left[\phi_{1}(l)-\phi_{2}(l)\right] d y\right| \\
& +\varrho\left|\phi_{1}(\eta)-\phi_{2}(\eta)\right| \\
& +\beta \mid \phi_{1}(\eta) \int_{0}^{h} f(\tau) g\left(\hat{\varphi}_{1}(\eta-c \tau)\right) d \tau-\phi_{1}(\eta) \int_{0}^{h} f(\tau) g\left(\hat{\varphi}_{2}(\eta-c \tau)\right) d \tau \\
& +\phi_{1}(\eta) \int_{0}^{h} f(\tau) g\left(\hat{\varphi}_{2}(\eta-c \tau)\right) d \tau-\phi_{2}(\eta) \int_{0}^{h} f(\tau) g\left(\hat{\varphi}_{2}(\eta-c \tau)\right) d \tau \mid \\
\leq & {\left[2 d_{1}+\varrho+\beta g\left(i_{0}\right)\right] \max _{y \in[-l, l]}\left|\phi_{1}(y)-\phi_{2}(y)\right| } \\
& +\beta s_{0} g^{\prime}(0) \max _{y \in[-l, l]}\left|\varphi_{1}(y)-\varphi_{2}(y)\right| .
\end{aligned}
$$


Then we can obtain the continuity of $\mathcal{F}_{1}$. Similarly, one can deduce the continuity of $\mathcal{F}_{2}$. The proof is completed.

By the definition of $\Gamma_{l}$, it is easy to see that $\Gamma_{l}$ is nonempty, bounded, closed and convex. Then based on Propositions 2.1, 2.2 and Schauder's fixed point theorem, we obtain the following theorem.

Theorem 2.1 There exists $\left(s_{l}(z), i_{l}(z)\right) \in \Gamma_{l}$ such that $\mathcal{F}\left(s_{l}, i_{l}\right)(z)=\left(s_{l}(z), i_{l}(z)\right)$, which satisfies

$$
\underline{s}(z) \leq s_{l}(z) \leq s_{0}, \quad \underline{i}(z) \leq i_{l}(z) \leq \bar{i}(z) \quad \text { for } z \in[-l, l] .
$$

\subsection{Existence of solutions of (1.7) on $\mathbb{R}$}

Choose a positive increasing constant sequence $\left\{l_{n}\right\}_{n=1}^{\infty}$ such that $l_{n}>\rho$ and $\lim _{n \rightarrow \infty} l_{n}=\infty$. Then by Theorem 2.1, we obtain that there exists some $\left(s_{l_{n}}, i_{l_{n}}\right) \in \Gamma_{l_{n}}$ such that

$$
\left\{\begin{aligned}
c s_{l_{n}}^{\prime}(z)= & d_{1} \int_{-\infty}^{\infty} J(y) \hat{s}_{l_{n}}(z-y) d y-d_{1} s_{l_{n}}(z) \\
& -\beta s_{l_{n}}(z) \int_{0}^{h} f(\tau) g\left(\hat{i}_{l_{n}}(z-c \tau)\right) d \tau, \\
c i_{l_{n}}^{\prime}(z)= & d_{2} \int_{-\infty}^{\infty} J(y) \hat{i}_{l_{n}}(z-y) d y+\beta s_{l_{n}}(z) \int_{0}^{h} f(\tau) g\left(\hat{i}_{l_{n}}(z-c \tau)\right) d \tau \\
& -\left(d_{2}+\gamma\right) i_{l_{n}}(z), \quad n \in \mathbb{N}^{*},
\end{aligned}\right.
$$

where

$$
\hat{s}_{l_{n}}(z)=\left\{\begin{array}{l}
s_{l_{n}}\left(l_{n}\right), \quad z>l_{n}, \\
s_{l_{n}}(z), \quad|z| \leq l_{n}, \\
\underline{s}(z), \quad z<-l_{n},
\end{array} \quad \hat{i}_{l_{n}}(z)=\left\{\begin{array}{l}
i_{l_{n}}\left(l_{n}\right), \quad z>l_{n}, \\
i_{l_{n}}(z), \quad|z| \leq l_{n}, \\
\underline{i}(z), \quad z<-l_{n},
\end{array}\right.\right.
$$

and

$$
\underline{s}(z) \leq s_{l_{n}}(z) \leq s_{0}, \quad \underline{i}(z) \leq i_{l_{n}}(z) \leq \bar{i}(z), \quad z \in\left[-l_{n}, l_{n}\right] .
$$

Inequalities (2.29) imply that $s_{l_{n}}(z)$ and $i_{l_{n}}(z)$ are all uniformly bounded on $z \in\left[-l_{n}, l_{n}\right]$, which together with (2.28) guarantees that $s_{l_{n}}^{\prime}(z)$ and $i_{l_{n}}^{\prime}(z)$ are all uniformly bounded on $z \in$ $\left[-l_{n}, l_{n}\right]$. Differentiating system (2.28), one can infer that $s_{l_{n}}^{\prime \prime}(z)$ and $i_{l_{n}}^{\prime \prime}(z)$ are all uniformly bounded on $z \in\left[-l_{n}+\rho, l_{n}-\rho\right]$. Applying Arzela-Ascoli theorem on $\left[-l_{n}+\rho, l_{n}-\rho\right]$ for every $n \in \mathbb{N}^{*}$ large enough, we have a subsequence which is still denoted by $l_{n}$ through the diagonal argument such that $\lim _{n \rightarrow \infty} l_{n}=\infty$ and

$$
s_{l_{n}} \rightarrow s, \quad i_{l_{n}} \rightarrow i, \quad s_{l_{n}}^{\prime} \rightarrow s^{\prime}, \quad i_{l_{n}}^{\prime} \rightarrow i^{\prime} \quad \text { as } n \rightarrow \infty,
$$

uniformly in any compact subinterval of $\mathbb{R}$. Furthermore, by Lebesgue dominated convergence theorem, we have that

$$
\lim _{n \rightarrow \infty} s_{l_{n}}(z) \int_{0}^{h} f(\tau) g\left(\hat{i}_{l_{n}}(z-c \tau)\right) d \tau=s(z) \int_{0}^{h} f(\tau) g(i(z-c \tau)) d \tau
$$




$$
\begin{aligned}
& \lim _{n \rightarrow \infty} \int_{-\infty}^{\infty} J(y) \hat{s}_{l_{n}}(z-y) d y=\int_{-\infty}^{\infty} J(y) s(z-y) d y, \\
& \lim _{n \rightarrow \infty} \int_{-\infty}^{\infty} J(y) \hat{i}_{l_{n}}(z-y) d y=\int_{-\infty}^{\infty} J(y) i(z-y) d y .
\end{aligned}
$$

Passing to the limits in (2.28) and (2.29) as $n \rightarrow \infty$, we obtain that

$$
\left\{\begin{aligned}
c s^{\prime}(z)= & d_{1} \int_{-\infty}^{\infty} J(y) s(z-y) d y-d_{1} s(z) \\
& -\beta s(z) \int_{0}^{h} f(\tau) g(i(z-c \tau)) d \tau \\
c i^{\prime}(z)= & d_{2} \int_{-\infty}^{\infty} J(y) i(z-y) d y+\beta s(z) \int_{0}^{h} f(\tau) g(i(z-c \tau)) d \tau \\
& -\left(d_{2}+\gamma\right) i(z)
\end{aligned}\right.
$$

and

$$
\underline{s}(z) \leq s(z) \leq s_{0}, \quad \underline{i}(z) \leq i(z) \leq \bar{i}(z), \quad z \in \mathbb{R} .
$$

Therefore, we have the following results.

Theorem 2.2 For $R_{0}>1$ and $c>c^{*}$, there exists some $(s(z), i(z)), z \in \mathbb{R}$ satisfying $(2.30)$ and (2.31). Furthermore,

$$
\|s\|_{C^{2}(\mathbb{R})}+\|i\|_{C^{2}(\mathbb{R})} \leq C_{0}
$$

with some $C_{0}$ independent on $l$.

\subsection{Asymptotic boundary of solution for (1.7)}

Lemma 2.6 The solution $(s(z), i(z))$ of (1.7) satisfies

(i) $s(-\infty)=s_{0}, i(-\infty)=0$ and $\lim _{z \rightarrow-\infty} e^{-\lambda_{1} z} i(z)=1$;

(ii) $0<\int_{\mathbb{R}} s(z) \int_{0}^{h} f(\tau) g(i(z-c \tau)) d \tau d z<\infty, \int_{\mathbb{R}} i(z) d z<\infty$ and $i(\infty)=0$;

(iii) $s(\infty)<s_{0}$ and $\beta \int_{\mathbb{R}} s(z) \int_{0}^{h} f(\tau) g(i(z-c \tau)) d \tau d z=\gamma \int_{\mathbb{R}} i(z) d z=c\left(s_{0}-s_{\infty}\right)$.

Proof The proof of (i). For $z \in \mathbb{R}$, it follows from (2.31) that

$$
s_{0}-\mu^{-1} e^{\mu z} \leq s(z) \leq s_{0} \quad \text { and } \quad e^{\lambda_{1} z}\left(1-M e^{\alpha z}\right) \leq i(z) \leq e^{\lambda_{1} z} .
$$

Applying the squeeze theorem in (2.32) gives that

$$
s(-\infty)=s_{0}, \quad i(-\infty)=0
$$

and

$$
\lim _{z \rightarrow-\infty} e^{-\lambda{ }^{1} z} i(z)=1
$$

This completes the proof of (i).

The proof of (ii). From (2.31), (A1) and the definitions of $\underline{s}(z), \underline{i}(z)$, we get

$$
\int_{-\infty}^{\infty} s(z) \int_{0}^{h} f(\tau) g(i(z-c \tau)) d \tau d z \geq \int_{-\infty}^{\infty} \underline{s}(z) \int_{0}^{h} f(\tau) g(\underline{i}(z-c \tau)) d \tau d z>0 .
$$


Note that

$$
\begin{aligned}
& \int_{\eta}^{x} \int_{-\infty}^{\infty} J(y)[s(z-y)-s(z)] d y d z \\
& \quad=-\int_{\eta}^{x} \int_{-\infty}^{\infty} J(y) y \int_{0}^{1} s^{\prime}(z-t y) d t d y d z \\
& \quad=\int_{-\infty}^{\infty} J(y) y \int_{0}^{1}[s(\eta-t y)-s(x-t y)] d t d y,
\end{aligned}
$$

then

$$
\begin{aligned}
\lim _{\eta \rightarrow-\infty} \int_{\eta}^{x} \int_{-\infty}^{\infty} J(y)[s(z-y)-s(z)] d y d z & =\int_{-\infty}^{\infty} J(y) y \int_{0}^{1}\left[s_{0}-s(x-t y)\right] d t d y \\
& =-\int_{-\infty}^{\infty} J(y) y \int_{0}^{1} s(x-t y) d t d y
\end{aligned}
$$

Consequently, one can deduce for $x \in \mathbb{R}$ that

$$
\left|\int_{-\infty}^{x} \int_{-\infty}^{\infty} J(y)[s(z-y)-s(z)] d y d z\right| \leq s_{0} \int_{-\infty}^{\infty} J(y)|y| d y:=\sigma_{0} .
$$

We can infer from the first equation in (1.7) that

$$
\begin{aligned}
& \beta \int_{-\infty}^{x} s(z) \int_{0}^{h} f(\tau) g(i(z-c \tau)) d \tau d z \\
& \quad=d_{1} \int_{-\infty}^{x} \int_{-\infty}^{\infty} J(y)[s(z-y)-s(z)] d y d z+c s_{0}-c s(x) \\
& \quad \leq d_{1} \sigma_{0}+c s_{0}
\end{aligned}
$$

which yields

$$
\int_{-\infty}^{\infty} s(z) \int_{0}^{h} f(\tau) g(i(z-c \tau)) d \tau d z<\infty
$$

Analogously, one can obtain

$$
\left|\int_{-\infty}^{\infty} \int_{-\infty}^{\infty} J(y)[i(z-y)-i(z)] d y d z\right| \leq i_{0} \int_{-\infty}^{\infty} J(y)|y| d y:=\sigma_{1} .
$$

Integrating the second equation in (1.7) over $\mathbb{R}$, we get

$$
\begin{aligned}
\gamma \int_{-\infty}^{\infty} i(z) d z \leq & d_{2} \int_{-\infty}^{\infty} \int_{-\infty}^{\infty} J(y)[i(z-y)-i(z)] d y d z \\
& +\beta \int_{-\infty}^{\infty} s(z) \int_{0}^{h} f(\tau) g(i(z-c \tau)) d \tau d z+c i_{0}<\infty
\end{aligned}
$$

It follows from (2.36) that $\int_{\mathbb{R}} i(z) d z<\infty$, which together with the boundedness of $i^{\prime}(z)$ implies that $i(\infty)=0$. This ends the proof of (ii). 
The proof of (iii). To obtain the existence of $s(\infty)$, we assume for the contrary that $\limsup _{z \rightarrow \infty} s(z)>\liminf _{z \rightarrow \infty} s(z)$. Then Fluctuation Lemma [45] implies that there is a sequence $\left\{z_{n}\right\}$ satisfying $z_{n} \rightarrow \infty$ as $n \rightarrow \infty$ such that

$$
\lim _{n \rightarrow \infty} s\left(z_{n}\right)=\limsup _{z \rightarrow \infty} s(z):=\sigma_{2} \quad \text { and } \quad s^{\prime}\left(z_{n}\right)=0
$$

Meanwhile, there exists a sequence $\left\{\eta_{n}\right\}$ satisfying $\eta_{n} \rightarrow \infty$ as $n \rightarrow \infty$ such that

$$
\lim _{n \rightarrow \infty} s\left(\eta_{n}\right)=\liminf _{z \rightarrow \infty} s(z):=\sigma_{3}<\sigma_{2} \quad \text { and } \quad s^{\prime}\left(\eta_{n}\right)=0
$$

From the first equation in (1.7), we have

$$
c s^{\prime}\left(z_{n}\right)=d_{1} \int_{-\infty}^{\infty} J(y)\left[s\left(z_{n}-y\right)-s\left(z_{n}\right)\right] d y d z-\beta s\left(z_{n}\right) \int_{0}^{h} f(\tau) g\left(i\left(z_{n}-c \tau\right)\right) d \tau .
$$

Letting $n \rightarrow \infty$ in (2.39) and using (2.37), $i(\infty)=0$ and $g(0)=0$, we obtain

$$
\lim _{n \rightarrow \infty} \int_{-\infty}^{\infty} J(y) s\left(z_{n}-y\right) d y=\lim _{n \rightarrow \infty} s\left(z_{n}\right)=\sigma_{2}
$$

Set

$$
s_{n}(y)=s\left(z_{n}-y\right) \text {. }
$$

Then we will show that $\lim _{n \rightarrow \infty} s_{n}(y) \rightarrow \sigma_{2}$ for any $y \in \operatorname{supp} J:=\Omega$. Take a sufficiently small $\tilde{\varepsilon}>0$ and let

$$
\Omega_{\tilde{\varepsilon}}=\Omega \cap\left\{y \in \Omega \mid \lim _{n \rightarrow \infty} s_{n}(y)<\sigma_{2}-\tilde{\varepsilon}\right\}
$$

From (2.40)-(2.42), one can get

$$
\begin{aligned}
\sigma_{2} & =\lim _{n \rightarrow \infty} \int_{\Omega} J(y) s\left(z_{n}-y\right) d y \\
& =\lim _{n \rightarrow \infty} \int_{\Omega} J(y) s_{n}(y) d y \\
& \leq \limsup _{n \rightarrow \infty} \int_{\Omega \backslash \Omega_{\tilde{\varepsilon}}} J(y) s_{n}(y) d y+\limsup _{n \rightarrow \infty} \int_{\Omega_{\tilde{\varepsilon}}} J(y) s_{n}(y) d y \\
& \leq \sigma_{2} \int_{\Omega \backslash \Omega_{\tilde{\varepsilon}}} J(y) d y+\left(\sigma_{2}-\tilde{\varepsilon}\right) \int_{\Omega_{\tilde{\varepsilon}}} J(y) d y \\
& =\sigma_{2}-\tilde{\varepsilon} \int_{\Omega_{\tilde{\varepsilon}}} J(y) d y,
\end{aligned}
$$

which implies that $m\left(\Omega_{\tilde{\varepsilon}}\right)=0$, where $m(\cdot)$ denotes the measure. Hence we obtain that $\lim _{n \rightarrow \infty} s_{n}(y)=\sigma_{2}$ almost everywhere in $\Omega$. However, $\left\{s_{n}\right\}$ is an equi-continuous family, so the convergence is everywhere in $\Omega$. That is,

$$
\lim _{n \rightarrow \infty} s_{n}(y)=\lim _{n \rightarrow \infty} s\left(z_{n}-y\right)=\sigma_{2}, \quad y \in \Omega .
$$


Analogously, using the similar arguments yields

$$
\lim _{n \rightarrow \infty} s\left(\eta_{n}-y\right)=\sigma_{3}<\sigma_{2}, \quad y \in \Omega .
$$

In a similar manner, we can show that

$$
\lim _{n \rightarrow \infty} s\left(z_{n}-t y\right)=\sigma_{2}, \quad t \in[0,1], y \in \Omega,
$$

and

$$
\lim _{n \rightarrow \infty} s\left(\eta_{n}-t y\right)=\sigma_{3}, \quad t \in[0,1], y \in \Omega
$$

Note that

$$
\lim _{n \rightarrow \infty} \int_{\eta_{n}}^{z_{n}} s(z) \int_{0}^{h} f(\tau) g(i(z-c \tau)) d \tau d z=0
$$

Integrating the first equation in (1.7) from $\eta_{n}$ to $z_{n}$ and using (2.43)-(2.45), we have that

$$
\begin{aligned}
0< & c\left(\sigma_{2}-\sigma_{3}\right) \\
= & c \lim _{n \rightarrow \infty}\left[s\left(z_{n}\right)-s\left(\eta_{n}\right)\right] \\
= & d_{1} \lim _{n \rightarrow \infty} \int_{\eta_{n}}^{z_{n}} \int_{-\infty}^{\infty} J(y)[s(z-y)-s(z)] d y d z \\
& -\beta \lim _{n \rightarrow \infty} \int_{\eta_{n}}^{z_{n}} s(z) \int_{0}^{h} f(\tau) g(i(z-c \tau)) d \tau d z \\
= & d_{1} \lim _{n \rightarrow \infty} \int_{\eta_{n}}^{z_{n}} \int_{-\infty}^{\infty} J(y)[s(z-y)-s(z)] d y d z \\
= & d_{1} \lim _{n \rightarrow \infty} \int_{\eta_{n}}^{z_{n}} \int_{-\infty}^{\infty} J(y)(-y) \int_{0}^{1} s^{\prime}(z-t y) d t d y d z \\
= & d_{1} \lim _{n \rightarrow \infty} \int_{-\infty}^{\infty} J(y) y \int_{0}^{1}\left[s\left(\eta_{n}-t y\right)-s\left(z_{n}-t y\right)\right] d t d y \\
= & 0,{ }_{0}
\end{aligned}
$$

which yields a contradiction. Thus we obtain

$$
\limsup _{z \rightarrow \infty} s(z)=\liminf _{z \rightarrow \infty} s(z)
$$

which implies that the limit $s(\infty)$ exists.

We next prove that $s_{\infty}<s_{0}$. Since $s(z) \leq s_{0}$, we have $s_{\infty} \leq s_{0}$. Assume that $s_{\infty}=s_{0}$, then we get

$$
s(-\infty)=s_{\infty}=s_{0} .
$$


Integrating the first equation in (1.7) from $-x$ to $x$ yields

$$
\begin{aligned}
c[s(x)-s(-x)]= & d_{1} \int_{-x}^{x} \int_{-\infty}^{\infty} J(y)[s(z-y)-s(z)] d y d z \\
& -\beta \int_{-x}^{x} s(z) \int_{0}^{h} f(\tau) g(i(z-c \tau)) d \tau d z \\
= & d_{1} \int_{-x}^{x} \int_{-\infty}^{\infty} J(y)(-y) \int_{0}^{1} s^{\prime}(z-t y) d t d y d z \\
& -\beta \int_{-x}^{x} s(z) \int_{0}^{h} f(\tau) g(i(z-c \tau)) d \tau d z \\
= & d_{1} \int_{-\infty}^{\infty} J(y)(-y) \int_{0}^{1}[s(x-t y)-s(-x-t y)] d t d y \\
& -\beta \int_{-x}^{x} s(z) \int_{0}^{h} f(\tau) g(i(z-c \tau)) d \tau d z .
\end{aligned}
$$

Letting $x \rightarrow \infty$ in (2.48) and using (2.47), we obtain

$$
\int_{-\infty}^{\infty} s(z) \int_{0}^{h} f(\tau) g(i(z-c \tau)) d \tau d z=0
$$

then a contradiction appears. Thus we have

$$
s_{\infty}<s_{0}
$$

Furthermore, integrating the first equation in (1.7) over $\mathbb{R}$ and applying Fubini theorem, we get

$$
\beta \int_{-\infty}^{\infty} s(z) \int_{0}^{h} f(\tau) g(i(z-c \tau)) d \tau d z=c\left(s_{0}-s_{\infty}\right)
$$

Integrating the second equation in (1.7) over $\mathbb{R}$ and applying Fubini theorem and $i( \pm \infty)=$ 0 , we deduce

$$
\beta \int_{-\infty}^{\infty} s(z) \int_{0}^{h} f(\tau) g(i(z-c \tau)) d \tau d z=\gamma \int_{-\infty}^{\infty} i(z) d z
$$

This ends the proof of (iii).

Therefore, we have obtained the following result.

Theorem 2.3 Suppose that $R_{0}>1$ and $c>c^{*}$. Then system (1.5) admits a nontrivial and nonnegative traveling wave solution $(s(x+c t), i(x+c t))$ satisfying (1.8). Furthermore, $\lim _{z \rightarrow-\infty} e^{-\lambda_{1} z} i(z)=1$ and

$$
\beta \int_{-\infty}^{\infty} s(z) \int_{0}^{h} f(\tau) g(i(z-c \tau)) d \tau d z=\gamma \int_{-\infty}^{\infty} i(z) d z=c\left(s_{0}-s_{\infty}\right)
$$




\section{Nonexistence of traveling wave solutions}

In this section, a detailed proof is provided for the nonexistence of traveling wave solutions of (1.5) in two cases: $R_{0}>1$ and $0<c<c^{*}$, as well as $R_{0} \leq 1$ and $c>0$. The main results of this section are stated in Theorems 3.1 and 3.2, respectively.

\subsection{Case 1: $R_{0}>1$ and $0<c<c^{*}$}

We present the main result of this case as follows.

Theorem 3.1 Suppose that $R_{0}>1$ and $0<c<c^{*}$. Then there exist no nontrivial and nonnegative traveling wave solutions $(s(x+c t), i(x+c t))$ of $(1.5)$ satisfying $i( \pm \infty)=0, s(-\infty)=s_{0}$ and $\sup _{z \in \mathbb{R}} s(z) \leq s_{0}$.

Proof The proof of this theorem is by contradiction. Assume that there exists a nontrivial and nonnegative traveling wave solution $(s(x+c t), i(x+c t))$ of system (1.5) satisfying $i( \pm \infty)=0, s(-\infty)=s_{0}$ and $\sup _{z \in \mathbb{R}} s(z) \leq s_{0}$.

Since $\lim _{z \rightarrow-\infty} s(z)=s_{0}$ and $\lim _{z \rightarrow-\infty} i(z)=0$, there exist $\hat{z}<0$ and sufficiently small constant $\delta_{0}>0$ such that

$$
s(z)>\frac{\beta s_{0} g^{\prime}(0)+\gamma}{2 \beta g^{\prime}(0)} \text { and } 0<i(z)<\delta_{0}
$$

for $z \leq \hat{z}$. Therefore, one can deduce from (2.12) and (3.1) that

$$
\begin{aligned}
c i^{\prime}(z)= & d_{2} \int_{-\infty}^{\infty} J(y)[i(z-y)-i(z)] d y \\
& +\beta s(z) \int_{0}^{h} f(\tau) g(i(z-c \tau)) d \tau-\gamma i(z) \\
\geq & d_{2} \int_{-\infty}^{\infty} J(y)[i(z-y)-i(z)] d y \\
& +\frac{\beta s_{0} g^{\prime}(0)+\gamma}{2 g^{\prime}(0)}\left[g^{\prime}(0)-\hat{\varepsilon}\right] \int_{0}^{h} f(\tau) i(z-c \tau) d \tau-\gamma i(z)
\end{aligned}
$$

for $z \leq \hat{z}$. Due to (3.2) holds for $\hat{\varepsilon} \in(0,1)$, one can get

$$
\begin{aligned}
c i^{\prime}(z) \geq & d_{2} \int_{-\infty}^{\infty} J(y)[i(z-y)-i(z)] d y \\
& +\frac{\beta s_{0} g^{\prime}(0)+\gamma}{2} \int_{0}^{h} f(\tau)[i(z-c \tau)-i(z)] d \tau+\frac{\beta s_{0} g^{\prime}(0)-\gamma}{2} i(z)
\end{aligned}
$$

for $z \leq \hat{z}$. By the integrability of $i(z)$, we define

$$
K(z):=\int_{-\infty}^{z} i(s) d s
$$


Integrating (3.3) from $-\infty$ to $z$ with $z \leq \hat{z}$, using (3.4) and $i(-\infty)=0$, we obtain that

$$
\begin{aligned}
& \frac{\beta s_{0} g^{\prime}(0)-\gamma}{2} K(z) \\
& \leq c i(z)-d_{2} \int_{-\infty}^{z} \int_{-\infty}^{\infty} J(y)[i(s-y)-i(s)] d y d s \\
& \quad+\frac{\beta s_{0} g^{\prime}(0)+\gamma}{2} \int_{-\infty}^{z} \int_{0}^{h} f(\tau)[i(s)-i(s-c \tau)] d \tau d s \\
& =c i(z)-d_{2} \int_{-\infty}^{z} \int_{-\infty}^{\infty} J(y)[i(s-y)-i(s)] d y d s \\
& \quad+\frac{\beta s_{0} g^{\prime}(0)+\gamma}{2} \int_{0}^{h} f(\tau) \int_{z-c \tau}^{z} i(s) d s d \tau .
\end{aligned}
$$

Applying Fubini theorem and (3.4) gives

$$
\begin{aligned}
\int_{-\infty}^{z} \int_{-\infty}^{\infty} J(y) i(s-y) d y d s & =\int_{-\infty}^{\infty} J(y) \int_{-\infty}^{z} i(s-y) d s d y \\
& =\int_{-\infty}^{\infty} J(y) \int_{-\infty}^{z-y} i(s) d s d y \\
& =\int_{-\infty}^{\infty} J(y) K(z-y) d y
\end{aligned}
$$

Substituting (3.6) into (3.5) yields

$$
\begin{aligned}
\frac{\beta s_{0} g^{\prime}(0)-\gamma}{2} K(z) \leq & c i(z)-d_{2} \int_{-\infty}^{\infty} J(y)[K(z-y)-K(z)] d y \\
& +\frac{\beta s_{0} g^{\prime}(0)+\gamma}{2} \int_{0}^{h} f(\tau) \int_{z-c \tau}^{z} i(s) d s d \tau .
\end{aligned}
$$

In view of

$$
\begin{aligned}
& \int_{-\infty}^{z} \int_{-\infty}^{\infty} J(y)[K(s-y)-K(s)] d y d s \\
& =\int_{-\infty}^{z} \int_{-\infty}^{\infty}(-y) J(y) \int_{0}^{1} K^{\prime}(s-\theta y) d \theta d y d s \\
& =\int_{-\infty}^{\infty}(-y) J(y) \int_{0}^{1} K(z-\theta y) d \theta d y
\end{aligned}
$$

and

$$
\begin{aligned}
& \int_{-\infty}^{z} \int_{0}^{h} f(\tau) \int_{x-c \tau}^{x} i(s) d s d \tau d x \\
& \quad=\int_{0}^{h} f(\tau)\left[\int_{-\infty}^{z-c \tau} \int_{s}^{s+c \tau} i(s) d x d s+\int_{z-c \tau}^{z} \int_{s}^{z} i(s) d x d s\right] d \tau \\
& \quad=\int_{0}^{h} f(\tau)\left[c \tau \int_{-\infty}^{z-c \tau} i(s) d s+\int_{z-c \tau}^{z} i(s)(z-s) d s\right] d \tau \\
& \quad \leq \int_{0}^{h} f(\tau)\left[c \tau \int_{-\infty}^{z-c \tau} i(s) d s+c \tau \int_{z-c \tau}^{z} i(s) d s\right] d \tau
\end{aligned}
$$




$$
\begin{aligned}
& =\operatorname{ch} \int_{-\infty}^{z} i(s) d s \\
& =\operatorname{chK}(z),
\end{aligned}
$$

we obtain that $J * K(\cdot)-K(\cdot)$ and $\int_{0}^{h} f(\tau) \int_{x-c \tau}^{x} i(s) d s d \tau$ are integrable on $(-\infty, z]$ for $z \leq \hat{z}$. Hence from (3.7)-(3.9), one derives that $K(\cdot)$ is integrable on $(-\infty, z]$ for $z \leq \hat{z}$.

Then integrating (3.7) from $-\infty$ to $z$ and using (3.8) and (3.9), we have

$$
\begin{aligned}
\frac{\beta s_{0} g^{\prime}(0)-\gamma}{2} \int_{-\infty}^{z} K(s) d s \leq & {\left[c+\frac{\beta s_{0} g^{\prime}(0)+\gamma}{2} c h\right] K(z) } \\
& +d_{2} \int_{-\infty}^{\infty} y J(y) \int_{0}^{1} K(z-\theta y) d \theta d y
\end{aligned}
$$

for $z \leq \hat{z}$. Since $y K(z-\theta y)$ is nonincreasing for $\theta \in[0,1]$,

$$
\begin{aligned}
\frac{\beta s_{0} g^{\prime}(0)-\gamma}{2} \int_{-\infty}^{z} K(s) d s & \leq\left[c+\frac{\beta s_{0} g^{\prime}(0)+\gamma}{2} c h+d_{2} \int_{-\infty}^{\infty} y J(y) d y\right] K(z) \\
& \leq\left[c+\frac{\beta s_{0} g^{\prime}(0)+\gamma}{2} c h\right] K(z)
\end{aligned}
$$

for $z \leq \hat{z}$. Due to $K(z)$ being nondecreasing with respect to $z$, for $\kappa>0$ there holds

$$
\frac{\beta s_{0} g^{\prime}(0)-\gamma}{2} \kappa K(z-\kappa) \leq\left[c+\frac{\beta s_{0} g^{\prime}(0)+\gamma}{2} c h\right] K(z) .
$$

Therefore, there exists a sufficiently large constant $\kappa_{0}$ and some $\epsilon \in(0,1)$ such that

$$
K\left(z-\kappa_{0}\right) \leq \epsilon K(z), \quad z \leq \hat{z},
$$

and

$$
\mu_{0}=\frac{1}{\kappa_{0}} \ln \frac{1}{\epsilon}>0
$$

Set

$$
Q(z):=K(z) e^{-\mu_{0} z},
$$

then using (3.11) and (3.12), we have that

$$
Q\left(z-\kappa_{0}\right)=K\left(z-\kappa_{0}\right) e^{-\mu_{0}\left(z-\kappa_{0}\right)} \leq \epsilon K(z) e^{-\mu_{0}\left(z-\kappa_{0}\right)}=Q(z),
$$

which together with the fact $Q(z) \geq 0$ implies that the limit $Q(z)$ at minus infinity exists. On the other hand, by (3.12) and (3.4), we get $\lim _{z \rightarrow \infty} Q(z)=0$. Hence there exists a positive constant $Q_{0}$ such that

$$
Q(z) \leq Q_{0}, \quad z \in \mathbb{R},
$$


namely,

$$
K(z) e^{-\mu_{0} z} \leq Q_{0}, \quad z \in \mathbb{R} .
$$

Moreover, we obtain from (3.13) and (A3) that

$$
\begin{aligned}
\int_{-\infty}^{\infty} J(y) K(z-y) d y e^{-\mu_{0} z} & =\int_{-\infty}^{\infty} J(y) e^{-\mu_{0} y} K(z-y) e^{-\mu_{0}(z-y)} d y \\
& \leq Q_{0} \int_{-\infty}^{\infty} J(y) e^{-\mu_{0} y} d y \\
& \leq M_{1}, \quad z \in \mathbb{R},
\end{aligned}
$$

where $M_{1}$ is a positive constant. By the second equation in (1.7), $s(z) \leq s_{0}$ and $g(i) \leq g^{\prime}(0) i$, we have that

$$
\begin{aligned}
c i^{\prime}(z)= & d_{2} \int_{-\infty}^{\infty} J(y) i(z-y) d y-d_{2} i(z) \\
& +\beta s(z) \int_{0}^{h} f(\tau) g(i(z-c \tau)) d \tau-\gamma i(z) \\
\leq & d_{2} \int_{-\infty}^{\infty} J(y) i(z-y) d y-d_{2} i(z) \\
& +\beta s_{0} g^{\prime}(0) \int_{0}^{h} f(\tau) i(z-c \tau) d \tau-\gamma i(z) .
\end{aligned}
$$

Integrating (3.15) from $-\infty$ to $z$ and using (3.4) and (3.6), we obtain that

$$
\begin{aligned}
c i(z) \leq & d_{2} \int_{-\infty}^{\infty} J(y) K(z-y) d y-d_{2} K(z) \\
& +\beta s_{0} g^{\prime}(0) \int_{-\infty}^{z} \int_{0}^{h} f(\tau) i(s-c \tau) d \tau d s-\gamma K(z) \\
= & d_{2} \int_{-\infty}^{\infty} J(y) K(z-y) d y-\left(d_{2}+\gamma\right) K(z) \\
& +\beta s_{0} g^{\prime}(0) \int_{0}^{h} f(\tau) \int_{-\infty}^{z} i(s-c \tau) d s d \tau \\
= & d_{2} \int_{-\infty}^{\infty} J(y) K(z-y) d y-\left(d_{2}+\gamma\right) K(z) \\
& +\beta s_{0} g^{\prime}(0) \int_{0}^{h} f(\tau) K(z-c \tau) d \tau \\
\leq & d_{2} \int_{-\infty}^{\infty} J(y) K(z-y) d y-\left(d_{2}+\gamma\right) K(z)+\beta s_{0} g^{\prime}(0) K(z)
\end{aligned}
$$

where the last inequality is induced by the monotonicity of $K(z)$. Utilizing (3.13), (3.14) and (3.16), one can get that there exists a constant $P_{1}>0$ such that

$$
i(z) e^{-\mu_{0} z} \leq P_{1}, \quad z \in \mathbb{R} .
$$


Noting (A3) and (3.17), there exists a positive constant $M_{2}$ such that

$$
\begin{aligned}
\int_{-\infty}^{\infty} J(y) i(z-y) d y e^{-\mu_{0} z} & =\int_{-\infty}^{\infty} J(y) i(z-y) e^{-\mu_{0} z} d y \\
& =\int_{-\infty}^{\infty} J(y) e^{-\mu_{0} y} i(z-y) e^{-\mu_{0}(z-y)} d y \\
& \leq P_{1} \int_{-\infty}^{\infty} J(y) e^{-\mu_{0} y} d y \leq M_{2}
\end{aligned}
$$

and

$$
\begin{aligned}
\int_{0}^{h} f(\tau) i(z-c \tau) e^{-\mu_{0} z} d \tau & =\int_{0}^{h} f(\tau) e^{-\mu_{0} c \tau} i(z-c \tau) e^{-\mu_{0}(z-c \tau)} d \tau \\
& \leq P_{1} \int_{0}^{h} f(\tau) e^{-\mu_{0} c \tau} d \tau \leq P_{1} .
\end{aligned}
$$

Then it follows from (3.15) and (3.17)-(3.19) that

$$
\sup _{z \in \mathbb{R}}\left\{i(z) e^{-\mu_{0} z}\right\}<\infty, \quad \sup _{z \in \mathbb{R}}\left\{i^{\prime}(z) e^{-\mu_{0} z}\right\}<\infty .
$$

Moreover, we get from the second equation of (1.7) that, for $z \in \mathbb{R}$,

$$
\begin{aligned}
& d_{2} \int_{-\infty}^{\infty} J(z) i(z-y) d y-d_{2} i(z)-c i^{\prime}(z) \\
& \quad+\beta s_{0} g^{\prime}(0) \int_{0}^{h} f(\tau) i(z-c \tau) d \tau-\gamma i(z) \\
& \quad=\beta s_{0} g^{\prime}(0) \int_{0}^{h} f(\tau) i(z-c \tau) d \tau-\beta s(z) \int_{0}^{h} f(\tau) g(i(z-c \tau)) d \tau .
\end{aligned}
$$

Since $s(-\infty)=s_{0}, i(-\infty)=0$, we have that for any $\varepsilon>0$, there exists a constant $z^{*} \ll 0$ such that

$$
s_{0} g^{\prime}(0)-\frac{s(z) g(i(z-c \tau))}{i(z-c \tau)}<\varepsilon \quad \text { for } z<z^{*}
$$

Then we get that

$$
\begin{aligned}
& s_{0} g^{\prime}(0) i(z-c \tau)-s(z) g(i(z-c \tau)) \\
& \quad=i(z-c \tau)\left[s_{0} g^{\prime}(0)-\frac{s(z) g(i(z-c \tau))}{i(z-c \tau)}\right] \\
& \quad \leq\left[\frac{s_{0} g^{\prime}(0)-\frac{s(z) g(i(z-c \tau))}{i(z-c \tau)}+i(z-c \tau)}{2}\right]^{2} \\
& \quad \leq\left[\frac{\varepsilon+i(z-c \tau)}{2}\right]^{2} \\
& \quad \leq i^{2}(z-c \tau) \quad \text { for } z<z^{*} .
\end{aligned}
$$


From (3.20) and (3.22), we obtain

$$
\sup _{z \in \mathbb{R}}\left\{e^{-2 \mu_{0} z}\left[\beta s_{0} g^{\prime}(0) \int_{0}^{h} f(\tau) i(z-c \tau) d \tau-\beta s(z) \int_{0}^{h} f(\tau) g(i(z-c \tau)) d \tau\right]\right\}<\infty
$$

Hence

$$
\beta \int_{-\infty}^{\infty} e^{-\lambda z}\left[s_{0} g^{\prime}(0) \int_{0}^{h} f(\tau) i(z-c \tau) d \tau-s(z) \int_{0}^{h} f(\tau) g(i(z-c \tau)) d \tau\right] d z<\infty
$$

for $0<\operatorname{Re} \lambda<2 \mu_{0}$.

For $\lambda \in \mathbb{C}$ with $0<\operatorname{Re} \lambda<\mu_{0}$, define the two-sided Laplace transform of $i(z)$ by

$$
\mathcal{L}(\lambda):=\int_{-\infty}^{\infty} i(z) e^{-\lambda z} d z
$$

In view of

$$
\int_{-\infty}^{\infty} e^{-\lambda z} \int_{-\infty}^{\infty} J(y) i(z-y) d y d z=\int_{-\infty}^{\infty} J(y) e^{-\lambda y} \mathcal{L}(\lambda) d y
$$

and taking two-sided Laplace transform on (3.21), we obtain

$$
\begin{aligned}
\Theta(\lambda, c) \mathcal{L}(\lambda)= & \beta \int_{-\infty}^{\infty} e^{-\lambda z}\left[s_{0} g^{\prime}(0) \int_{0}^{h} f(\tau) i(z-c \tau) d \tau\right. \\
& \left.-s(z) \int_{0}^{h} f(\tau) g(i(z-c \tau)) d \tau\right] d z
\end{aligned}
$$

for $\lambda \in \mathbb{C}$ with $0<\operatorname{Re} \lambda<\mu_{0}$, where $\Theta(\lambda, c)$ is defined in (2.2).

The property of Laplace transform [43] claims that either there exists a real number $\mu_{0}$ such that $L(\lambda)$ is analytic for $\lambda \in \mathbb{C}$ with $0<\operatorname{Re} \lambda<\mu_{0}$ and $\lambda=\mu_{0}$ is singular point of $L(\lambda)$, or for $\lambda \in \mathbb{C}$ with $\operatorname{Re} \lambda>0, L(\lambda)$ is well defined. As for (3.25), the two Laplace integrals can be analytically continued to the whole right half-plane. Otherwise the integral on the left-hand side of (3.25) has a singularity at $\lambda=\mu_{0}$ and it is analytic for all $\lambda$ with $\operatorname{Re} \lambda<\mu_{0}$. However, (3.24) implies that the integral on the right-hand side of (3.25) is actually analytic for all $\lambda$ with $\operatorname{Re} \lambda<2 \mu_{0}$, a contradiction. Thus we obtain that (3.25) holds for all $\lambda$ with $\operatorname{Re} \lambda>0$. However, Lemma 2.1 implies that $\Delta(\infty, c)=\infty$ for $c \in\left(0, c^{*}\right)$. A contradiction occurs. The proof of this theorem is completed.

\subsection{Case 2: $R_{0} \leq 1$ and $c>0$}

We give the main result of this case as follows.

Theorem 3.2 Suppose that $R_{0} \leq 1$ and $c>0$. Then there exist no nontrivial and nonnegative traveling wave solutions $(s(x+c t), i(x+c t))$ of $(1.5)$ satisfying $i( \pm \infty)=0, s(-\infty)=s_{0}$ and $\sup _{z \in \mathbb{R}} s(z) \leq s_{0}$.

Proof Suppose to the contrary that there exists a traveling wave solution $(s(x+c t), i(x+c t))$ of (1.5) satisfying $i( \pm \infty)=0, s(-\infty)=s_{0}$ and $\sup _{z \in \mathbb{R}} s(z) \leq s_{0}$. We prove the desired results in two subcases. 
Subcase 1. $R_{0}<1$, i.e., $\beta s_{0} g^{\prime}(0) / \gamma<1$.

Integrating the second equation in (1.7) over $\mathbb{R}$ yields

$$
\begin{aligned}
c \int_{-\infty}^{\infty} i^{\prime}(z) d z= & d_{2} \int_{-\infty}^{\infty} \int_{-\infty}^{\infty} J(y) i(z-y) d y d z-\left(d_{2}+\gamma\right) \int_{-\infty}^{\infty} i(z) d z \\
& +\beta \int_{-\infty}^{\infty} s(z) \int_{0}^{h} f(\tau) g(i(z-c \tau)) d \tau d z .
\end{aligned}
$$

Since $i( \pm \infty)=0$ and $s(z) \leq s_{0}$, one can obtain from (3.26) that

$$
\begin{aligned}
\left(d_{2}+\gamma\right) \int_{-\infty}^{\infty} i(z) d z \leq & d_{2} \int_{-\infty}^{\infty} \int_{-\infty}^{\infty} J(y) i(z-y) d y d z \\
& +\beta s_{0} g^{\prime}(0) \int_{-\infty}^{\infty} \int_{0}^{h} f(\tau) i(z-c \tau) d \tau d z
\end{aligned}
$$

Applying Fubini theorem in (3.27), we have that

$$
\begin{aligned}
\left(d_{2}+\gamma\right) \int_{-\infty}^{\infty} i(z) d z \leq & d_{2} \int_{-\infty}^{\infty} J(y) \int_{-\infty}^{\infty} i(z-y) d z d y \\
& +\beta s_{0} g^{\prime}(0) \int_{0}^{h} f(\tau) \int_{-\infty}^{\infty} i(z-c \tau) d z d \tau \\
= & d_{2} \int_{-\infty}^{\infty} i(z) d z+\beta s_{0} g^{\prime}(0) \int_{-\infty}^{\infty} i(z) d z
\end{aligned}
$$

that is,

$$
\int_{-\infty}^{\infty} i(z) d z \leq \frac{\beta s_{0} g^{\prime}(0)}{\gamma} \int_{-\infty}^{\infty} i(z) d z<\int_{-\infty}^{\infty} i(z) d z
$$

which leads to a contradiction.

Subcase 2. $R_{0}=1$, i.e., $\beta s_{0} g^{\prime}(0)=\gamma$.

From the second equation in (1.7), we get that

$$
\begin{aligned}
c i^{\prime}(z)= & d_{2} \int_{-\infty}^{\infty} J(z-y)[i(y)-i(z)] d y \\
& +\beta s(z) \int_{0}^{h} f(\tau) g(i(z-c \tau)) d \tau-\gamma i(z) \\
= & d_{2} \int_{-\infty}^{\infty} J(z-y)[i(y)-i(z)] d y \\
& +\beta s(z) \int_{0}^{h} f(\tau) g(i(z-c \tau)) d \tau-\beta s_{0} g^{\prime}(0) i(z) .
\end{aligned}
$$


Integrating (3.28) over $\mathbb{R}$ yields

$$
\begin{aligned}
c \int_{-\infty}^{\infty} i^{\prime}(z) d z= & d_{2} \int_{-\infty}^{\infty} \int_{-\infty}^{\infty} J(z-y)[i(y)-i(z)] d y d z \\
& +\beta \int_{-\infty}^{\infty} s(z) \int_{0}^{h} f(\tau) g(i(z-c \tau)) d \tau d z \\
& -\beta s_{0} g^{\prime}(0) \int_{-\infty}^{\infty} i(z) d z
\end{aligned}
$$

Using the facts $i( \pm \infty)=0$ and Fubini theorem, we obtain from (3.29) that

$$
\begin{aligned}
0 & =\beta \int_{0}^{h} f(\tau) \int_{-\infty}^{\infty} s(z) g(i(z-c \tau)) d z d \tau-\beta s_{0} g^{\prime}(0) \int_{-\infty}^{\infty} i(z) d z \\
& =\beta \int_{0}^{h} f(\tau) \int_{-\infty}^{\infty} s(z+c \tau) g(i(z)) d z d \tau-\beta s_{0} g^{\prime}(0) \int_{0}^{h} f(\tau) \int_{-\infty}^{\infty} i(z) d z d \tau \\
& \leq \beta g^{\prime}(0) \int_{0}^{h} f(\tau) \int_{-\infty}^{\infty}\left[s(z+c \tau)-s_{0}\right] i(z) d z d \tau .
\end{aligned}
$$

Due to $\left(s(z+c \tau)-s_{0}\right) i(z) \leq 0$ for $z \in \mathbb{R}, \tau \in[0, h]$ and the continuity of $s(z)$ and $i(z)$, it follows from (3.30) that

$$
\left[s(z+c \tau)-s_{0}\right] i(z)=0
$$

Since the interior of supp $i$ is not empty, we have from (3.31) that

$$
s(z+c \tau)=s_{0}, \quad z \in \operatorname{supp} i, \tau \in[0, h] .
$$

For $z \in \operatorname{supp} i, \tau \in[0, h]$, by the translation invariance of traveling wave solutions, we get from the first equation in (1.7) that

$$
\begin{aligned}
c s^{\prime}(z+c \tau)= & d_{1} \int_{-\infty}^{\infty} J(y) s(z-y+c \tau) d y-d_{1} s(z+c \tau) \\
& -\beta s(z+c \tau) \int_{0}^{h} f(\tau) g(i(z)) d \tau .
\end{aligned}
$$

Using (3.32) and (3.33), we then have

$$
\begin{aligned}
0 & =d_{1} \int_{-\infty}^{\infty} J(y)\left[s(z+c \tau-y)-s_{0}\right] d y-\beta s_{0} \int_{0}^{h} f(\tau) g(i(z)) d \tau \\
& \leq-\beta s_{0} g(i(z))<0, \quad z \in \operatorname{supp} i, \tau \in[0, h]
\end{aligned}
$$

which yields another contradiction. The proof of this theorem is finished. 


\section{Application and conclusion}

In this section, we will give a typical example to support our abstract results. The choice of $g(i)=\frac{i}{1+\alpha i}(\alpha>0$ is a coefficient $)$ in (1.5) leads to

$$
\left\{\begin{aligned}
s_{t}(x, t)= & d_{1} \int_{-\infty}^{\infty} J(x-y)[s(y, t)-s(x, t)] d y \\
& -\beta s(x, t) \int_{0}^{h} f(\tau) \frac{i(x, t-\tau)}{1+\alpha i(x, t-\tau)} d \tau \\
i_{t}(x, t)= & d_{2} \int_{-\infty}^{\infty} J(x-y)[i(y, t)-i(x, t)] d y \\
& +\beta s(x, t) \int_{0}^{h} f(\tau) \frac{i(x, t-\tau)}{1+\alpha i(x, t-\tau)} d \tau-\gamma i(x, t) .
\end{aligned}\right.
$$

Obviously, it is easy to verify that $g(i)=\frac{i}{1+\alpha i}$ satisfies assumptions (A1) and (A2). Applying Theorems 2.3, 3.1 and 3.2, we obtain the following result.

\section{Theorem 4.1 There exists a positive constant $c^{*}$ such that}

(i) If $R_{0}=\beta s_{0} / \gamma>1$ and $c>c^{*}$, then system (4.1) admits a nontrivial and nonnegative traveling wave solution $(s(x+c t), i(x+c t))$ satisfying $s(\infty)=s_{\infty}<s_{0}=s(-\infty)$, $i( \pm \infty)=0$;

(ii) If $R_{0}>1$ and $0<c<c^{*}$ or $R_{0} \leq 1$ and $c>0$, then system (4.1) admits no nontrivial and nonnegative traveling wave solutions $(s(x+c t), i(x+c t))$ satisfying $i( \pm \infty)=0$, $s(-\infty)=s_{0}$ and $\sup _{z \in \mathbb{R}} s(z) \leq s_{0}$.

It is known that traveling wave solutions of nonlinear partial differential equations have been extensively studied due to significant applications in many fields [1, 2, 33, 34, 37, 55 57]. In the present paper, we have studied the traveling wave solutions of a nonlocal diffusion epidemic model with nonlinear incidences and distributed delay. It has been found that the existence and nonexistence of traveling wave solutions are totally determined by the basic reproduction number and the minimum wave speed $c^{*}$. More precisely, if $R_{0}>1$ and $c>c^{*}$, then (1.5) has a nontrivial and nonnegative traveling wave solution satisfying (1.8); if $0<c<c^{*}$ or $R_{0} \leq 1$, there exist no such traveling wave solutions. In particular, we give an example with the choice of $g(i)=\frac{i}{1+\alpha i}(\alpha>0)$ to support our theoretical analysis. It is noted that the existence and nonexistence of traveling wave solutions can indicate whether or not the disease spreads; if it spreads, the wave speed can illustrate the spreading speed. Results on this topic may help one predict how fast a disease invades geographically, and accordingly, take necessary measures in advance to prevent the disease, or at least decrease possible negative consequences.

To explore the effect of the diffusive rate $d_{2}$ of the infective individuals on the minimum wave speed $c^{*}$, we use (2.2) and Lemma 2.1 to get

$$
\Theta_{c^{*}}\left(\lambda^{*}, c^{*}\right)=-\lambda^{*}-\lambda^{*} \beta s_{0} g^{\prime}(0) \int_{0}^{h} \tau f(\tau) e^{-\lambda^{*} c^{*} \tau} d \tau<0
$$

and

$$
\Theta_{d_{2}}\left(\lambda^{*}, c^{*}\right)=\int_{-\infty}^{\infty} J(y)\left(e^{-\lambda^{*} y}-1\right) d y \geq-\lambda^{*} \int_{-\infty}^{\infty} y J(y) d y=0 .
$$

Combining (4.2) and (4.3), we obtain the fact that the geographical movement of infective individuals can increase the speed of the spread of disease. 
There are a few problems deserving further investigation, for instance, time periodic traveling wave solutions, the uniqueness and stability of traveling wave solution in (1.5).

\begin{abstract}
Acknowledgements
We would like to thank Dr. Jingdong Wei and Dr. Jiangbo Zhou for some helpful suggestions. Meanwhile, we are deeply grateful to the anonymous referees for the valuable suggestions and comments which have greatly improved the presentation of our paper.
\end{abstract}

\title{
Funding
}

This work was supported by the Innovation Project for Graduate Student Research of Jiangsu Province [grant number KYLX15_1073] and the China Postdoctoral Science Foundation [grant number 2018M642174].

\section{Competing interests}

The authors declare that they have no competing interests.

\section{Authors' contributions}

All authors contributed to the draft of the manuscript, all authors read and approved the final manuscript.

\section{Publisher's Note}

Springer Nature remains neutral with regard to jurisdictional claims in published maps and institutional affiliations.

\section{Received: 26 December 2018 Accepted: 6 March 2019 Published online: 15 March 2019}

\section{References}

1. Ali, A., Seadawy, A., Lu, D.: Soliton solutions of the nonlinear Schrödinger equation with dual power law nonlinearity and resonant nonlinear Schrödinger equation and their modulation instability analysis. Optik 145, 79-88 (2017)

2. Arshad, M., Seadawy, A.R., Lu, D.: Exact bright-dark solitary wave solutions of the higher-order cubic-quintic nonlinear Schrödinger equation and its stability. Optik 138, 40-49 (2017)

3. Bai, Z., Zhang, S.: Traveling waves of a diffusive SIR epidemic model with a class of nonlinear incidence rates and distributed delay. Commun. Nonlinear Sci. Numer. Simul. 22,1370-1381 (2015)

4. Bates, P.W., Chen, F.: Spectral analysis and multidimensional stability of traveling waves for nonlocal Allen-Cahn equation. J. Math. Anal. Appl. 273, 45-57 (2002)

5. Bates, P.W., Fife, P.C., Ren, X., Wang, X.: Traveling waves in a convolution model for phase transitions. Arch. Ration. Mech. Anal. 138, 105-136 (1997)

6. Beretta, E., Takeuchi, Y.: Global stability of an SIR epidemic model with time delays. J. Math. Biol. 33, $250-260$ (1995)

7. Capasso, V., Serio, G.: A generalization of the Kermack-McKendrick deterministic epidemic model. Math. Biosci. 42, 43-61 (1978)

8. Carr, J., Chmaj, A.: Uniqueness of travelling waves for nonlocal monostable equations. Proc. Am. Math. Soc. 132 2433-2439 (2004)

9. Chen, F.: Stability and uniqueness of traveling waves for system of nonlocal evolution equations with bistable nonlinearity. Discrete Contin. Dyn. Syst. 24, 659-673 (2009)

10. Chen, X.: Existence, uniqueness and asymptotic stability of travelling waves in non-local evolution equations. Adv. Differ. Equ. 2, 125-160 (1997)

11. Coville, J., Davila, J., Martinez, S.: Nonlocal anisotropic dispersal with monostable nonlinearity. J. Differ. Equ. 244, 3080-3118 (2008)

12. Coville, J., Davila, J., Martinez, S.: Pulsating fronts for nonlocal dispersion and KPP nonlinearity. Ann. Inst. Henri Poincaré Nonl. Anal. 30, 179-223 (2013)

13. Coville, J., Dupaigne, L.: On a nonlocal reaction diffusion equation arising in population dynamics. Proc. R. Soc. Edinb. Sect. A 137, 727-755 (2007)

14. Diekmann, O., Kaper, $\mathrm{H} .:$ On the bounded solutions of a nonlinear convolution equation. Nonlinear Anal. 2, 721-737 (1978)

15. Ducrot, A., Magal, P.: Travelling wave solutions for an infection-age structured model with diffusion. Proc. R. Soc. Edinb., Sect. A 139, 459-482 (2009)

16. Ducrot, A., Magal, P., Ruan, S.: Travelling wave solutions in multigroup age-structure epidemic models. Arch. Ration. Mech. Anal. 195, 311-331 (2010)

17. Enatsua, Y., Nakata, Y., Muroya, Y.: Lyapunov functional techniques for the global stability analysis of a delayed SIRS epidemic model. Nonlinear Anal., Real World Appl. 13, 2120-2133 (2010)

18. Hosono, Y., llyas, B.: Traveling waves for a simple diffusive epidemic model. Math. Models Methods Appl. Sci. 5, 935-966 (1995)

19. Ignat, L., Rossi, J.D.: A nonlocal convolution-diffusion equation. J. Funct. Anal. 251, 399-437 (2007)

20. Ignat, L., Rossi, J.D.: Decay estimates for nonlocal problems via energy methods. J. Math. Pures Appl. 92, 163-187 (2009)

21. Kermack, W.O., McKendrick, A.G.: Contribution to the mathematical theory of epidemics. Proc. R. Soc. Lond. B 115 700-721 (1927)

22. Kermack, W.O., McKendrick, A.G.: Contribution to the mathematical theory of epidemics, Part II. Proc. R. Soc. Lond. B $138,55-83$ (1932)

23. Kermack, W.O., McKendrick, A.G.: Contribution to the mathematical theory of epidemics, Part III. Proc. R. Soc. Lond. B 141, 94-112 (1933) 
24. Korobeinikov, A.: Lyapunov functions and global stability for SIR and SIRS epidemiological models with non-linear transmission. Bull. Math. Biol. 68, 615-626 (2006)

25. Korobeinikov, A.: Global properties of infectious disease models with nonlinear incidence. Bull. Math. Biol. 69 , 1871-1886 (2007)

26. Korobeinikov, A., Maini, P.K.: Nonlinear incidence and stability of infectious disease models. Math. Med. Biol. 22, 113-128 (2005)

27. Kuang, Y.: Delay Differential Equations with Applications in Population Dynamics. Academic Press, San Diego (1993)

28. Li, W., Sun, Y., Wang, Z.: Entire solutions in the Fisher-KPP equation with nonlocal dispersal. Nonlinear Anal., Real World Appl. 11, 2302-2313 (2010)

29. Li, Y., Li, W., Lin, G.: Traveling waves of a delayed diffusive SIR epidemic model. Commun. Pure Appl. Anal. 14, $1001-1022(2015)$

30. Li, Y., Li, W., Yang, F.: Traveling waves for a nonlocal dispersal SIR model with delay and external supplies. Appl. Math. Comput. 247, 723-740 (2014)

31. Liu, W., Hethcote, H.W., Levin, S.A.: Dynamical behavior of epidemiological models with nonlinear incidence rates. J. Math. Biol. 25, 359-380 (1987)

32. Liu, W., Levin, S.A., Iwasa, Y.: Influence of nonlinear incidence rates upon the behavior of SIRS epidemiological models. J. Math. Biol. 23, 187-204 (1986)

33. Lu, D., Seadawy, A., Arshad, M.: Applications of extended simple equation method on unstable nonlinear Schrödinger equations. Optik, Int. J. Light Electron Opt. 140, 136-144 (2017)

34. Lu, D., Seadawy, A., Arshad, M.: Bright-dark solitary wave and elliptic function solutions of unstable nonlinear Schrödinger equation and their applications. Opt. Quantum Electron. 50, 23 (2018)

35. Pan, S.: Traveling wave fronts in an epidemic model with nonlocal diffusion and time delay. Int. J. Math. Anal. 2 1083-1088 (2008)

36. Pan, S., Li, W., Lin, G.: Travelling wave fronts in nonlocal reaction-diffusion systems and applications. Z. Angew. Math. Phys. 60, 377-392 (2009)

37. Seadawy, A.R., Lu, D.: Bright and dark solitary wave soliton solutions for the generalized higher order nonlinear Schrödinger equation and its stability. Results Phys. 7, 43-48 (2017)

38. Sun, Y., Li, W., Wang, Z.: Entire solutions in nonlocal dispersal equations with bistable nonlinearity. J. Differ. Equ. 251 551-581 (2011)

39. Takeuchi, Y., Ma, W., Beretta, E.: Global asymptotic properties of a delay SIR epidemic model with finite incubation times. Nonlinear Anal. 42, 931-947 (2000)

40. Wang, X.: Metastability and stability of patterns in a convolution model for phase transitions. J. Differ. Equ. 183, 434-461 (2002)

41. Wang, Z., Wu, J.: Traveling waves of a diffusive Kermack-McKendrick epidemic model with nonlocal delayed transmission. Proc. R. Soc. A 466, 237-261 (2010)

42. Wang, Z., Wu, J., Liu, R.: Traveling waves of avian influenza spread. Proc. Am. Math. Soc. 140, 3931-3946 (2012)

43. Widder, D.V.: The Laplace Transform. Princeton University Press, Princeton (1941)

44. Wu, J.: Theory and Applications of Partial Functional Differential Equations. Springer, New York (1996)

45. Wu, J., Zou, X.: Traveling wave fronts of reaction-diffusion systems with delay. J. Dyn. Differ. Equ. 13, 651-687 (2001)

46. Xu, Z:: Traveling waves in a Kermack-McKendrick epidemic model with diffusion and latent period. Nonlinear Anal. $111,66-81(2014)$

47. Yagisita, $H .:$ Existence and nonexistence of traveling waves for a nonlocal monostable equation. Publ. Res. Inst. Math. Sci. 45, 925-953 (2009)

48. Yagisita, H.: Existence of traveling wave solutions for a nonlocal bistable equation: an abstract approach. Publ. Res. Inst. Math. Sci. 45, 955-979 (2009)

49. Yang, F., Li, W., Wang, Z.: Traveling waves in a nonlocal dispersal SIR epidemic model. Nonlinear Anal., Real World Appl. 23, 129-147 (2015)

50. Yang, F., Li, Y., Li, W., Wang, Z.: Traveling waves in a nonlocal dispersal Kermack-McKendrick epidemic model. Discrete Contin. Dyn. Syst., Ser. B 18, 1969-1993 (2013)

51. Zhang, G.: Global stability of wavefronts with minimal speeds for nonlocal dispersal equations with degenerate nonlinearity. Nonlinear Anal. 74, 6518-6529 (2011)

52. Zhang, G., Li, W., Wang, Z.: Spreading speeds and traveling waves for nonlocal dispersal equations with degenerate monostable nonlinearity. J. Differ. Equ. 252, 5096-5124 (2012)

53. Zhang, L.: Existence, uniqueness and exponential stability of traveling wave solutions of some integral differential equations arising from neural networks. J. Differ. Equ. 197, 162-196 (2004)

54. Zhang, T., Wang, W.: Existence of traveling wave solutions for influenza model with treatment. J. Math. Anal. Appl. 419, 469-495 (2014)

55. Zhen, Z., Wei, J., Tian, L., Zhou, Z., Chen, W.: Wave propagation in a diffusive SIR epidemic model with spatio-temporal delay. Math. Methods Appl. Sci. 0, 1-25 (2018)

56. Zhen, Z., Wei, J., Zhou, J., Tian, L.: Wave propagation in a nonlocal diffusion epidemic model with nonlocal delayed effects. Appl. Math. Comput. 339, 15-37 (2018)

57. Zhou, J., Xu, J., Wei, J., Xu, H.: Existence and non-existence of traveling wave solutions for a nonlocal dispersal SIR epidemic model with nonlinear incidence rate. Nonlinear Anal., Real World Appl. 41, 204-231 (2018) 